\title{
Herman Cappelen und die Frage nach der philosophischen Relevanz begrifflicher Wahrheiten
}

\section{$1 \quad$ Einleitung}

Ich bin im Kapitel 2 des vorliegenden Buchs bereits auf den Umstand eingegangen, dass manche Philosophinnen und Philosophen einen engen $\mathrm{Zu}$ sammenhang zwischen der Begriffsanalyse, der Methode möglicher Fälle und Intuitionen über mögliche Fälle annehmen. Deshalb werden Argumente gegen die Aussagekraft von Intuitionen oft auch als Argumente gegen die Begriffsanalyse aufgefasst. Der wichtigste zeitgenössische Kritiker von Intuitionen, der sich gleichzeitig als Kritiker der Begriffsanalyse versteht, ist Herman Cappelen.

In Philosophy without Intuitions (2012) argumentiert Cappelen gegen die Auffassung, dass Intuitionen bei der Begründung von Theorien in der zeitgenössischen analytischen Philosophie eine wichtige Rolle spielen. Die spezifische Auffassung, die er kritisiert, bezeichnet er als »Centrality«:

Centrality (of Intuitions in Contemporary Philosophy): Contemporary analytic philosophers rely on intuitions as evidence (or as a source of evidence) for philosophical theories. ${ }^{1}$

Die Idee, dass Intuitionen für die zeitgenössische analytische Philosophie von zentraler Bedeutung sind, liegt unter anderem deshalb nahe, weil die Wörter »intuition« und »intuitive(-ly)« in den Texten der Philosophinnen und Philosophen dieser Tradition häufig auftreten. Mit diesem Umstand setzt sich Cappelen im Teil I seines Buchs auseinander. Er versucht, zu zeigen, dass mit »intuition « und »intuitive(-ly)« normalerweise weder im gewöhnlichen Englisch noch im »Philosophen-Englisch« auf eine besondere Art der Evidenz Bezug genommen wird. Cappelen identifiziert verschiedene Strategien zur Paraphrase der Rede von Intuitionen in philosophischen Texten, anhand derer deutlich werden sollte, dass die Verwendung des Intuitions-Vokabulars durch Philosophinnen und Philosophen nicht belegt, dass irgendwelche mentalen Zustände oder Ereignisse mit dem Namen »Intuitionen « wesentlich in die Begründung philosophischer Thesen involviert sind. Je nach Kontext,

1 Cappelen 2012, S. 3. 
so Cappelen, können Vorkommnisse von »Intuitively, $p$ « oder ähnlichen Formulierungen zum Beispiel wie folgt paraphrasiert werden:

- Man könnte auf den ersten Blick meinen, dass $p$.

- Eine einfache, aber nicht ganz akkurate Art, die Sachlage aufzufassen, wäre $p$.

- Für den Zweck der vorliegenden Abhandlung wird $p$ vorausgesetzt.

- Die meisten Leute sind sich einig, dass $p$.

$-p$.

Keiner dieser Sätze verweist auf eine besondere Art der Evidenz dafür, dass $p-$ oder jedenfalls nicht auf eine Evidenz von der Art, wie sie den Anhängerinnen und Anhängern von Intuitionen vorschwebt. Also gilt dies auch für »Intuitively, $p$ « in solchen Kontexten, in denen dieser Satz auf eine der genannten Weisen paraphrasiert werden kann.

Im Teil II von Philosophy without Intuitions wendet sich Cappelen von der Verwendung von Intuitions-Vokabular in der Philosophie ab und setzt sich mit der Idee auseinander, dass die Berufung auf Intuitionen aus philosophischen Texten implizit hervorgeht und sich insbesondere im Gebrauch der Methode möglicher Fälle manifestiert. Cappelen gibt drei Merkmale an, die ihm zufolge von den meisten Verteidigerinnen und Verteidigern von Intuitionen als charakteristisch für intuitive Urteile aufgefasst werden, und versucht, zu zeigen, dass Urteile mit diesen Merkmalen in der analytischen Gegenwartsphilosophie keine entscheidende Rolle spielen. Zu diesem Zweck bespricht er eine Reihe von bekannten philosophischen Argumenten, die besonders aussichtsreiche Kandidaten für Argumente zu sein scheinen, in denen auf Intuitionen zurückgegriffen wird. Viele davon sind Beispiele für die Anwendung der Methode möglicher Fälle. In Bezug auf sämtliche besprochenen Argumente verteidigt Cappelen die folgende Auffassung: Sollten irgendwelche Urteile, die in diesen Argumenten vorkommen, eines oder mehrere der drei für intuitive Urteile charakteristischen Merkmale aufweisen, spielt es jedenfalls für die Argumente keine Rolle, dass die betreffenden Urteile diese Merkmale aufweisen. Die drei Merkmale haben, mit Cappelen gesprochen, kein »wirksames Vorkommen « (effective occurrence) in philosophischen Argumenten. ${ }^{2}$

In der vorliegenden Abhandlung wird eine Philosophie-Konzeption verteidigt, die keine Berufung auf Intuitionen involviert. Dafür, dass Cappelens Buch darin dennoch diskutiert wird, gibt es mehrere Gründe. Der erste und wichtigste Grund besteht darin, dass eines der drei Merkmale, die Cappelen als charakteristisch für intuitive Urteile behandelt, das Merkmal ist, ausschließlich

2 Vgl. Cappelen 2012, S. 115f. 
auf Sprach- oder Begriffskompetenz zu beruhen. ${ }^{3}$ Und Cappelen versucht nicht nur zu zeigen, dass sich Philosophinnen und Philosophen in der Regel nicht auf Urteile berufen, die alle drei von ihm angeführten Merkmale aufweisen, sondern für die stärkere These, dass sich Philosophinnen und Philosophen üblicherweise nicht auf Urteile berufen, die auch nur eines dieser Merkmale aufweisen - oder dass zumindest keines der drei Merkmale in philosophischen Argumenten ein wirksames Vorkommen hat. ${ }^{4}$ Philosophinnen und Philosophen stützen sich also im für Cappelen relevanten Sinn ihm zufolge unter anderem auch nicht auf Urteile ab, die ausschließlich auf Sprachoder Begriffskompetenz beruhen. Am stärksten bringt er diese Auffassung zum Ausdruck, wenn er auf S. 202 schreibt: »[E]ven if there are conceptual a priori truths, exemplified by >All vixens are female foxes<, those are irrelevant to philosophical practice.« Und dass diese These von Cappelen relevant ist für die vorliegende Abhandlung, dürfte offensichtlich sein.

Ein zweiter Grund dafür, in der vorliegenden Abhandlung auf Cappelens Buch einzugehen, ist, dass er darin ein ganzes Kapitel dem Thema Begriffsanalyse widmet: Cappelen argumentiert in seinem Kapitel 10, das die Überschrift »Conceptual Analysis and Intuitions « trägt, für die These, dass es keinen Grund dafür gibt, weshalb sich Philosophinnen und Philosophen besonders für begriffliche Wahrheiten interessieren sollten, und stellt außerdem die folgende Behauptung auf:

Recognition of the marginal (or non-existent) role of intuitions in philosophical practice goes hand in hand with recognition of the marginal (or non-existent) role of anything reasonably labeled 'conceptual analysis' in philosophy. ${ }^{5}$

Mit der vorliegenden Abhandlung steht zum einen die These im Widerspruch, dass die Begriffsanalyse in der Philosophie nur eine marginale oder überhaupt keine Rolle spielt, und zum anderen die Idee, dass die philosophische Irrelevanz von Intuitionen irgendwelche Rückschlüsse auf die Relevanz der Begriffsanalyse zulässt.

Ein letzter Berührungspunkt zwischen Philosophy without Intuitions und dem Thema der vorliegenden Abhandlung ergibt sich daraus, dass Cappelen sich in seinem Buch mehrfach zur Auffassung bekennt, dass es keine begrifflichen Wahrheiten gibt. ${ }^{6}$ Da er die Argumente, die ihn von dieser Auffassung überzeugt haben, nicht diskutiert, sondern auf Williamsons The Philosophy

3 Vgl. Cappelen 2012, S. 113.

4 Vgl. z. B. Cappelen 2012, S. 114, 131.

5 Cappelen 2012, S. 206.

6 Vgl. Cappelen 2012, S. 178, 201, 211. 
of Philosophy verweist, ${ }^{7}$ wird auf diesen Punkt im vorliegenden Kapitel nicht weiter eingegangen.

Das Kapitel ist wie folgt aufgebaut: Im Abschnitt 2 gehe ich auf die Eigenschaften ein, die Cappelen als charakteristische Merkmale von Intuitionen identifiziert. Im Abschnitt 3 versuche ich, zu bestimmen, was Cappelen damit meint, dass ein Intuitions-Merkmal in einem Argument ein wirksames Vorkommen hat, und wie er begründet, dass die Frage nach dem wirksamen Vorkommen von Intuitions-Merkmalen für sein Projekt relevant ist. Ich werde dafür argumentieren, dass Cappelens Ausführungen in diesem Zusammenhang mit einer ganzen Reihe von Problemen einhergehen und dass letztlich noch nicht einmal ganz klar aus Cappelens Buch hervorgeht, was es bedeutet, dass ein Merkmal ein wirksames Vorkommen hat. Im Abschnitt 4 geht es um Cappelens Fallstudien und sein Bestreben, zu zeigen, dass in keinem der von ihm untersuchten Fälle eine Berufung auf eine begriffliche Wahrheit stattfindet. Ich liefere zuerst eine Übersicht über die verschiedenen Argumente, die Cappelen im Rahmen der Fallstudien zum Ausschluss einer Abstützung auf begriffliche Wahrheiten anführt. Wie sich zeigt, ist ein Teil dieser Argumente schon an und für sich und unabhängig von den konkreten Fällen, auf die Cappelen sie anwendet, nicht stichhaltig. Dann kritisiere ich die restlichen Argumente beziehungsweise die Art und Weise, wie Cappelen sie auf spezifische Fälle anwendet. Im Abschnitt 5 gehe ich auf Cappelens Begründung dafür ein, sich in seinem Buch nicht mit Gettier-Fällen auseinanderzusetzen. In diesem Kontext befasse ich mich auch mit seiner Behauptung, dass philosophische Fragen im Allgemeinen mit einem Anspruch auf Rechtfertigung einhergehen, und mit der Relevanz dieser Behauptung für die Frage nach der Rolle von Intuitionen in der Philosophie. Der Abschnitt 6 handelt schließlich von Cappelens Kapitel zum Thema Begriffsanalyse und seinem Versuch, zu zeigen, dass Philosophinnen und Philosophen, die dafür plädieren, Begriffe zu analysieren, vor eine Reihe von Herausforderungen gestellt sind, die sich kaum überwinden lassen.

\section{Intuitions-Merkmale}

Die drei Merkmale (features; daher die Abkürzung $\mathrm{F} \ll$ ) intuitiver Urteile, anhand derer implizite Bezugnahmen auf Intuitionen in philosophischen Texten erkannt werden sollen, sind die folgenden:

7 Vgl. Cappelen 2012, S. 210f. 
F1 Besondere Phänomenologie: Das Fällen eines intuitiven Urteils soll mit einer ganz spezifischen Empfindung einhergehen. ${ }^{8}$ Cappelen zitiert in diesem Zusammenhang unter anderem John Pollock und Alvin Plantinga:

Logically intuiting something is a phenomenologically unique experience which, although it may not be analyzable into other more familiar kinds of experience, is nevertheless a kind of experience a person can be quickly taught to recognize and label. ${ }^{9}$

... that peculiar sort of phenomenology with which we are well acquainted, but which I can't describe in any way other than as the phenomenology that goes with seeing that such a proposition is true..$^{10}$

F2 Epistemischer Fundaments-Status: Intuitive Urteile bedürfen keiner Begründung, können aber zur Begründung anderer Urteile herangezogen werden. ${ }^{11}$ Intuitive Urteile sind weder auf Wahrnehmung noch auf Erinnerungen oder die Berichte anderer Leute abgestützt und sind auch nicht aus Urteilen, die so abgestützt sind, hergeleitet. ${ }^{12}$

F3 Begrifflich wahr: Vielen Verteidigern von Intuitionen zufolge, so Cappelen, sind intuitive Urteile ausschließlich auf Sprach- oder Begriffskompetenz abgestützt. ${ }^{13}$

Es könnte der Anschein erweckt werden, dass F2 und F3 inkompatibel sind: Intuitive Urteile sollen den Status von »letzten Begründungen « aufweisen (F2), scheinen aber gemäß $\mathrm{F}_{3}$ dennoch auch ihrerseits begründbar zu sein, nämlich mit Bezug auf unsere Sprach- oder Begriffskompetenz. Diese Spannung lässt sich allerdings unabhängig von Cappelen (dieser geht auf diesen Punkt nicht ein, meint aber ohnehin, dass sich das Merkmal $F_{3}$ nicht zufriedenstellend ausbuchstabieren lasse ${ }^{14}$ ) für die Zwecke der vorliegenden Abhandlung leicht beheben: Dass ein Urteil auf unsere Sprach- oder Begriffskompetenz abgestützt ist, bedeutet nicht, dass wir es mit Bezug auf diese Kompetenz begründen können. Beantworten wir die Frage »Wieso meinst du, dass ein Junggeselle ein unverheirateter Mann ist? « zum Beispiel inspiriert von $P U \S 381$ mit »Ich habe Deutsch gelernt«, so liefern wir damit nicht eine Begründung dafür, dass ein Junggeselle ein unverheirateter Mann ist, und rechtfertigen auch nicht unsere diesbezügliche Überzeugung.

\footnotetext{
8 Vgl. Cappelen 2012, S. 101, 112, 117.

9 Pollock 1974, S. 321.

$10 \quad$ Plantinga 1993, S. 106.

11 Vgl. Cappelen 2012, S. 112.

12 Vgl. Cappelen 2012, S. 105, 112.

13 Vgl. Cappelen 2012, S. 113.

14 Vgl. Cappelen 2012, S. 126, Fußnote 11.
} 
Dazu gezwungen, das Urteil, dass ein Junggeselle ein unverheirateter Mann ist, zu begründen, würden wir vielleicht sagen, dass dies die Definition von »Junggeselle«sei, aberdamithätten wir das Urteil nichtauf ein fundamentaleres oder weniger begründungsbedürftiges Urteil zurückgeführt. Das Urteil, dass ein Junggeselle ein unverheirateter Mann ist, bedarf schon seinerseits keiner Begründung, und wir fällen es auf der Grundlage unserer Sprachkompetenz. $\mathrm{F}_{2}$ und $\mathrm{F}_{3}$ sind somit vereinbar.

Nicht nur sind F2 und $F_{3}$ vereinbar, sondern dass ein Urteil F3 aufweist, kann sogar der Grund dafür sein, dass es F2 aufweist. Cappelen schreibt selbst, dass die Vertreterinnen und Vertreter der Auffassung, dass bestimmte Propositionen F2-Status haben, davon ausgehen, dass diese Propositionen dieses Merkmal aufgrund von bestimmten anderen Eigenschaften aufweisen. ${ }^{15}$ Und begrifflich wahr zu sein, wäre wohl eine der Eigenschaften eines Urteils (vielleicht sogar die einzige), die der Eigenschaft zugrunde liegen kann, keiner Begründung zu bedürfen. ${ }^{16}$

\section{Wirksames Vorkommen}

\subsection{Definition}

Nach der Einführung der oben genannten drei Merkmale intuitiver Urteile streicht Cappelen heraus, dass nur ein wirksames Vorkommen von F1, F2 und F3 für die von ihm abgelehnte Auffassung sprechen würde, dass die Abstützung auf Intuitionen ein charakteristischer Wesenszug der gegenwärtigen analytischen Philosophie darstellt. Cappelen erklärt, was er mit dem wirksamen Vorkommen eines Intuitions-Merkmals meint, und kommt im Kapitel 8 seines Buchs im Rahmen der Besprechung konkreter philosophischer Argumente immer wieder auf diese Bedingung zurück. Seine Ausführungen in diesem Kontext sind allerdings nicht unproblematisch. Zum einen geht, wie weiter unten dargelegt werden soll, nicht eindeutig aus ihnen hervor, was es bedeutet, dass ein Merkmal ein wirksames Vorkommen in einem philosophischen Argument hat, zum anderen ist aber auch unklar, weshalb Cappelen von der Rolle, die Intuitionen in philosophischen Argumenten spielen, plötzlich übergeht zur Rolle, die Intuitions-Merkmale in philosophischen Argumenten spielen. Dass eine bestimmte Art von Entitäten, sagen wir $F$ s, in einem bestimmten Kontext

\footnotetext{
15 Vgl. Cappelen 2012, S. 124.

16 Ich lasse hier offen, dass ein Urteil begrifflich wahr sein und trotzdem einer Begründung bedürfen kann. Vgl. die Ausführungen zu synoptischen begrifflichen Wahrheiten im Kapitel 1 des vorliegenden Buchs.
} 
eine wichtige Rolle spielt, heißt nicht automatisch, dass auch die einzelnen Merkmale, die für das Fallen unter $F$ notwendig sind, im betreffenden Kontext eine wichtige Rolle spielen. Auch auf diesen Punkt gehe ich weiter unten näher ein.

Kommen wir aber zunächst darauf zurück, wie Cappelen den Begriff des wirksamen Vorkommens eines Merkmals einführt. Cappelen illustriert seine Idee zunächst anhand von drei Szenarien, die ich nachfolgend (in derselben Reihenfolge, in der sie bei Cappelen vorkommen) leicht vereinfacht wiedergebe.

(a) Nehmen wir an, ein bestimmtes Urteil $u$ weise F2 auf und bedürfe also keiner Begründung. Und nehmen wir weiter an, $u$ komme in den Erwägungen eines Philosophen vor, der sich dessen nicht bewusst ist, dass $u$ F2 aufweist. Er behandelt das Urteil $u$ zum Beispiel so, als bedürfe es eines empirischen Beweises. In diesem Fall ist zwar ein IntuitionsMerkmal in eine philosophische Erwägung involviert, aber sein Vorkommen ist nicht wirksam. Dass Intuitions-Merkmale auf diese Weise in philosophische Erwägungen eingehen, so Cappelen, spricht nicht für die von ihm abgelehnte These, dass die Abstützung auf Intuitionen in der gegenwärtigen analytischen Philosophie eine wichtige Rolle spielt. ${ }^{17}$

(b) Auf der anderen Seite könnte sich herausstellen, dass in Wirklichkeit kein Urteil begrifflich wahr ist, also $\mathrm{F}_{3}$ aufweist, ohne dass damit gezeigt wäre, dass Philosophinnen und Philosophen ihre Zeit nicht mit der Suche nach begrifflichen Wahrheiten verbringen. Denn dass es keine F3-Urteile gibt, ist damit vereinbar, dass die meisten Philosophinnen und Philosophen davon ausgehen, dass es sie doch gibt. ${ }^{18}$

(c) Nehmen wir an, das Fällen eines bestimmten Urteils $u^{\prime}$ gehe mit der speziellen Intuitions-Phänomenologie F1 einher und spiele in philosophischen Argumenten eine wichtige Rolle. Der Umstand, dass $u^{\prime}$ diese Phänomenologie aufweist, habe allerdings keinen Einfluss auf die Argumentation der Philosophinnen und Philosophen, in deren Argumenten das Urteil vorkommt. Das mit $u^{\prime}$ verbundene Gefühl würde von ihnen als unangenehmes Jucken abgetan. ${ }^{19}$ In diesem Fall, wäre ein Urteil mit F1 in der Philosophie relevant, aber das Merkmal F1 hätte kein wirksames Vorkommen. ${ }^{20}$

17 Vgl. Cappelen 2012, S. 115

18 Vgl. Cappelen 2012, S. 115f.

19 Diese Schilderung ist natürlich polemisch. Angesichts dessen, wie die Anhänger der Auffassung, dass Intuitionen mit einem speziellen Gefühl einhergehen, dieses Gefühl beschreiben, wäre es nicht möglich, es mit einem Jucken zu verwechseln (oder es überhaupt als etwas anderes zu interpretieren als das »Sich-wahr-Anfühlen« eines Urteils).

Vgl. Cappelen 2012, S. 116 . 
Mindestens die ersten beiden angeführten Szenarien werfen gewisse Fragen auf. Zunächst zu (a): Cappelen schreibt nicht, dass sich der Philosoph in diesem Szenario im Rahmen seiner philosophischen Erwägungen auf das F2-Urteil beruft, sondern nur, dass es in seinen Erwägungen vorkommt. Sollte sich der Philosoph nicht auf das F2-Urteil berufen, sondern es beispielsweise nur nebenbei erwähnen, ist offensichtlich, dass dieses Vorkommen eines F2Urteils nicht die These stützt, dass es in der Philosophie gängig ist, sich auf Intuitionen zu berufen. Angesichts von Cappelens weiterer Argumentation wird allerdings deutlich, dass Folgendes gemeint ist: Wenn sich ein Philosoph auf ein Urteil beruft, das das Merkmal F2 aufweist, ohne dass sich der Philosoph dieses besonderen Status des Urteils bewusst ist, und wenn der Philosoph beispielweise mit Bezug auf kontingente Tatsachen dafür argumentiert, dass das Urteil wahr ist, dann hat das Merkmal F2 im Argument dieses Philosophen kein wirksames Vorkommen.

Im Zusammenhang mit dem Szenario (b), das ja ebenfalls der Veranschaulichung davon dienen soll, was mit dem wirksamen Vorkommen eines Intuitions-Merkmals gemeint ist, ist besonders auffällig, dass darin gar nicht explizit vom wirksamen Vorkommen eines Intuitions-Merkmals die Rede ist. Vermutlich soll die betreffende Passage andeuten, dass das Merkmal, begrifflich wahr zu sein, ein wirksames Vorkommen haben kann, ohne instantiiert zu sein. Wir sollten Cappelen aber sicherlich nicht so verstehen, dass er sagt, dass es für das wirksame Vorkommen eines Intuitions-Merkmals hinreichend ist, dass ein Philosoph oder eine Philosophin nach Urteilen mit diesem Merkmal sucht. Denn aus Cappelens Fallstudien geht hervor, dass beispielsweise David Chalmers nach eigenen Angaben in der Tat unter anderem mit der Suche nach begrifflichen Wahrheiten befasst ist, und Cappelen meint trotzdem, in Chalmers Argumenten habe das Merkmal F3 kein wirksames Vorkommen. ${ }^{21}$ Auf Cappelens Argumentation in diesem Kontext komme ich weiter unten zurück.

Einen Versuch einer allgemeinen Beschreibung davon, unter welchen Bedingungen ein Intuitions-Merkmal ein wirksames Vorkommen hat, könnte man im Lichte der bisherigen Rekonstruktion von Cappelens Ausführungen wie folgt beginnen: Es ist eine notwendige Bedingung für das wirksame Vorkommen eines Intuitions-Merkmals in einem philosophischen Argument, dass die betreffende Philosophin oder der Philosoph ein Urteil, auf das sie oder er sich beruft, so behandelt, als weise es das betreffende Merkmal auf. Was es bedeutet, ein Urteil so zu behandeln, als weise es ein bestimmtes Merkmal auf, ist am einfachsten mit Bezug auf das Merkmal F2 ersichtlich, um welches es

Vgl. Cappelen 2012, S. $186 f$. 
im Szenario (a) ja auch geht: Ein Urteil so zu behandeln, als bedürfe es keiner Begründung, heißt, es nicht zu begründen, nicht zu behaupten, dass es einer Begründung bedürfe, und keine Gründe dafür zu suchen; im vorliegenden Kontext insbesondere keine empirischen Gründe. (Das ist natürlich nicht ohne Weiteres korrekt: Dass eine Behauptung keiner Begründung bedarf, bedeutet ja nicht, dass sie sich nicht begründen lässt; ${ }^{22}$ also sollte die Behandlung eines Urteils als nicht begründungsbedürftig damit vereinbar sein, es zu begründen. Diese Komplikation ignoriere ich allerdings für den Moment.)

Was bedeutet es, ein Urteil so zu behandeln, als habe es das IntuitionsMerkmal F3 und sei also begrifflich wahr? Dies ist nicht so leicht zu sagen. Eine Antwort auf die Frage könnte aber beispielsweise lauten, dass man keine empirischen Gründe für das Urteil sucht oder angibt und dass man es unterlässt, die Negation des Urteils als Option in Erwägung zu ziehen beziehungsweise Zweifel an der Wahrheit des Urteils zum Ausdruck zu bringen. ${ }^{23}$

Am schwierigsten dürfte es sein, zu bestimmen, was es heißt, ein Urteil so zu behandeln, als weise es das Intuitions-Merkmal F1, also die besondere Phänomenologie auf. Solange wir keinen Zusammenhang zwischen dem Vorliegen von F1 und dem Vorliegen der anderen Intuitions-Merkmale voraussetzen, ist nicht klar, weshalb der Umstand, dass ein Urteil F1 aufweist, uns dazu veranlassen sollte, dass Urteil in irgendeiner Hinsicht anders als jedes andere Urteil zu behandeln. In Betracht kommen würde allenfalls eine bestimmte verbale »Behandlung «, nämlich beim Anführen des Urteils zu erwähnen, dass es eine besondere Phänomenologie aufweist. ${ }^{24}$ Wir müssen allerdings im Blick behalten, dass wir hier von einer notwendigen Bedingung für das wirksame Vorkommen eines Intuitions-Merkmals sprechen und damit nach Cappelen letztlich auch von einer notwendigen Bedingung für die Berufung auf Intuitionen: F1 hat, sofern die bisherige Rekonstruktion von Cappelens Argumentation korrekt ist, kein wirksames Vorkommen in einem philosophischen Argument, wenn die betreffende Philosophin oder der Philosoph kein Urteil, auf das sie oder er sich beruft, so behandelt, als weise es F1 auf. Und die explizite Erwähnung der Phänomenologie eines Urteils zur notwendigen Bedingung für die Berufung auf eine Intuition zu machen, scheint

22 Auf diesen Umstand macht auch Cappelen aufmerksam. Vgl. Cappelen 2012, S. $161 f$.

23 Diese Bedingungen für die Behandlung eines Urteils als $\mathrm{F}_{3}$-Urteil klingen auch in Cappelen 2012 an (S. 136f., 179 bzw. S. 159f., 165, 177). Der Aspekt des In-Zweifel-Ziehens eines Urteils bzw. des In-Erwägung-Ziehens seiner Negation kommt bei Cappelen allerdings eher im Zusammenhang mit F2 als im Zusammenhang mit F3 zur Sprache.

Vgl. Cappelen 2012, S. 118. 
unangemessen zu sein - auch wenn Cappelen bisweilen den Eindruck macht, als würde er dies anders sehen. ${ }^{25}$

Welche anderen Bedingungen für das wirksame Vorkommen von IntuitionsMerkmalen können wir Cappelens Beurteilung der Szenarien (a) bis (c) entnehmen? Cappelens Beurteilung des Szenarios (b) können wir, wie bereits weiter oben angedeutet, weder eine notwendige noch eine hinreichende Bedingung zum wirksamen Vorkommen von Intuitions-Merkmalen entnehmen. Die einzige Erkenntnis, die wir daraus ziehen können, besteht darin, dass das Vorliegen eines Urteils, welches tatsächlich eines der drei Merkmale F1 bis F3 aufweist, keine notwendige Bedingung für das wirksame Vorkommen dieser Merkmale ist.

Aus Cappelens Ausführungen zum Szenario (c) können wir schließen, dass Intuitions-Merkmale, um ein wirksames Vorkommen zu haben, einen Einfluss auf die Argumentation von Philosophinnen und Philosophen haben müssen. Dass sie einen solchen Einfluss haben, kann sich theoretisch darin manifestieren, dass eine Philosophin explizit betont, dass sie ein bestimmtes Urteil deshalb besonders ernst nimmt, weil es eine besondere Phänomenologie aufweist, nicht begründungsbedürftig oder begrifflich wahr ist. ${ }^{26}$ Derartige Bemerkungen kommen in philosophischen Texten natürlich außerordentlich selten vor. Mindestens zwei Stellen in Cappelen 2012 legen außerdem nahe, dass Cappelen das Auftreten einer Bemerkung dieser Art nicht als hinreichend für das wirksame Vorkommen eines Intuitions-Merkmals erachtet: Damit ein Intuitions-Merkmal wirksam vorkommt, so anscheinend Cappelens Idee, muss ein Philosoph oder eine Philosophin sich nicht nur in seiner oder ihrer Argumentation auf ein Urteil berufen, von dem er oder sie annimmt, dass es $\mathrm{F}_{1}, \mathrm{~F}_{2}$ oder $\mathrm{F}_{3}$ aufweist, und den Umstand, dass das Urteil dieses Merkmal aufweist, als epistemisch relevant betrachten. Sondern es muss auch die Bedingung erfüllt sein, dass das fragliche Argument ohne die Annahme, dass das betreffende Urteil das fragliche Intuitions-Merkmal aufweist, seinen Zweck nicht erfüllen kann. Wenn das Argument auch solche Personen überzeugen kann, die im Gegensatz zu seinem Urheber oder seiner Urheberin nicht annehmen, dass das betreffende Urteil F1, F2 oder F3 aufweist, hat das entsprechende Intuitions-Merkmal in dem Argument kein wirksames Vorkommen. ${ }^{27}$ Diese Bedingung halte ich aus Gründen, auf die ich im Abschnitt 4 eingehen werde, für inadäquat.

25 Vgl. z. B. Cappelen 2012, S. 136, 138, 144, 166, 178.

26 Vgl. z. B. Cappelen 2012, S. 186.

27 Vgl. Cappelen 2012, S. 169, Fußnote 20, S. $186 f$. 
Als provisorische Definition davon, was »wirksames Vorkommen« bei Cappelen bedeutet, können wir festhalten:

Wirksames Vorkommen: Damit ein Intuitions-Merkmal in einem philosophischen Argument ein wirksames Vorkommen hat, muss der Philosoph oder die Philosophin, von dem oder der das Argument stammt, erstens ein bestimmtes Urteil, auf das er oder sie sich beruft, so behandeln, als weise es F1, F2 oder F3 auf. Und zweitens muss die Überzeugungskraft des Arguments an die Annahme gebunden sein, dass eines der involvierten Urteile F1, F2 oder F3 aufweist.

\subsection{Legitimation der Bedingung des wirksamen Vorkommens}

Cappelen bringt die Bedingung des wirksamen Vorkommens immer wieder in Verbindung mit dem Umstand, dass er die empirische Frage beantworten will, wie Philosophinnen und Philosophen tatsächlich vorgehen und wie sie tatsächlich argumentieren, und sich in seiner Monographie weder für die modale Frage interessiert, wie sie vorgehen oder argumentieren könnten, noch für die normative Frage, wie sie vorgehen oder argumentieren sollten. ${ }^{28} \mathrm{Im}$ Zusammenhang mit dem Intuitions-Merkmal F2 befasst sich Cappelen zum Beispiel mit dem oben bereits genannten Punkt, dass der Umstand, dass ein Urteil ausführlich begründet werde, damit vereinbar sei, dass das Urteil keiner Begründung bedürfe. Dazu schreibt Cappelen:

One difficulty with this proposal is that it is in tension with the emphasis on effective occurrences of F1-F3. As emphasized above, for the purposes of evaluating Centrality, ${ }^{29}$ presence of $\mathrm{F} 2$ that does not move and influence philosophical practice is uninteresting. Hypotheses about how philosophical practice could be or ought to be done won't tell us what philosophical practice is really like (if those possibilities are not realized and the practice isn't the way it ought to be). In particular, suppose the claim that $p$ is debated by philosophers: reasons are given and evidence is presented. $P$ is accepted or rejected based on the strength of those reasons and that evidence. Then the fact that it could be endorsed without justification constitutes no support for Centrality. ${ }^{30}$

28 Vgl. z. B. Cappelen 2012, S. 115. Die Behauptung, dass für ihn die modale Frage nicht von Bedeutung ist, steht prima facie in einem Spannungsverhältnis zur im letzten Absatz erwähnten Relevanz der Frage, ob ein Argument auch dann überzeugend wäre, wenn ein bestimmtes darin vorkommendes Urteil entgegen den Angaben des Urhebers oder der Urheberin des Arguments keines der drei Intuitions-Merkmale aufweisen würde. Wie ich weiter unten zu zeigen versuche, ist Cappelens Argumentation im Zusammenhang mit der Bedingung, dass ein Argument nicht überzeugend sein dürfe ohne die Annahme, dass eines der involvierten Urteile F1, F2 oder F3 aufweise, aber auch für sich allein schon problematisch.

29 Für eine Erklärung davon, worauf Cappelen mit »Centrality« Bezug nimmt, vgl. S. 213 des vorliegenden Kapitels.

30 Cappelen 2012, S. 119f. 
Ob Philosophinnen und Philosophen ein Urteil, das sie ausführlich begründen, ebenso gut unbegründet anführen könnten, sei für Cappelens Zwecke nicht relevant, da er sich dafür interessiere, wie PhilosophInnen tatsächlich vorgehen, und nicht dafür, wie sie vorgehen könnten oder sollten. Diese Entgegnung von Cappelen ist nicht zufriedenstellend. Mit $\mathrm{F}_{2}$ führt Cappelen nun einmal selbst eine modale Eigenschaft als eine der relevanten Eigenschaften von Intuitionen ein: die Eigenschaft, nicht begründet werden zu müssen. Damit macht er nolens volens auch die Frage, ob sich PhilosophInnen auf Intuitionen abstützen, zu einer partiell modalen Frage, denn zu ihrer Beantwortung müssen wir unter anderem die Frage beantworten, ob sich PhilosophInnen auf Urteile abstützen, die nicht begründet werden müssen. Und die Behauptung, dass diese letzte Frage darauf gerichtet ist, wie PhilosophInnen vorgehen könnten, statt auf den für Cappelen relevanten Punkt, wie PhilosophInnen tatsächlich vorgehen, ist irreführend: Wir können nach wie vor die Frage, ob PhilosophInnen sich tatsächlich auf Urteile abstützen, die nicht begründet werden müssen, von der Frage unterscheiden, ob PhilosophInnen sich auf Urteile abstützen könnten, die nicht begründet werden müssen. Und ob sich PhilosophInnen tatsächlich auf Urteile abstützen, die nicht begründet werden müssen, ist eine Frage über die tatsächliche philosophische Praxis.

Meines Erachtens bringt die Diskussion um die Wirksamkeit des Vorkommens von Intuitions-Merkmalen (und ihre im vorangehenden Absatz kritisierte Vermengung mit der Unterscheidung zwischen empirischen, modalen und normativen Fragen) Cappelens ganze Argumentation in Verwirrung: Cappelens Ausgangsfrage besteht darin, ob Intuitionen und (für die vorliegende Abhandlung relevanter) begriffliche Wahrheiten für philosophische Argumente von besonderer Relevanz sind. Immer, wenn es in seinen Fallstudien interessant wird - zum Beispiel, wenn der Eindruck entsteht, dass ein Philosoph oder eine Philosophin sich tatsächlich auf eine begriffliche Wahrheit beruft - lenkt er die Diskussion vom Vorkommen des betreffenden Intuitions-Merkmals um in Richtung von dessen wirksamem Vorkommen, begründet aber an keiner Stelle zufriedenstellend, weshalb die Anforderung des wirksamen Vorkommens legitim sein sollte.

Ich sehe ein, dass es für Cappelens Beurteilung der Centrality-These, also der These, dass zeitgenössische analytische Philosophinnen und Philosophen sich auf Intuitionen als Evidenzen (oder Quellen von Evidenz) für philosophische Theorien abstützen, ${ }^{31}$ relevant ist, ob Intuitionen in philosophischen Argumenten ein wirksames Vorkommen haben. Und zwar in dem Sinn, dass Centrality nur dann stimmt, wenn die entsprechenden Argumente nicht auch

Vgl. S. 213 des vorliegenden Kapitels. 
funktionieren würden ohne das Heranziehen von Intuitionen (also nach Cappelens Interpretation ohne das Heranziehen von Urteilen mit den Eigenschaften F1, F2 und F3). Nicht unmittelbar einsichtig ist, weshalb das wirksame Vorkommen von Intuitions-Merkmalen für die Beurteilung von Centrality relevant sein soll. Die Idee ist vermutlich, dass Centrality nur dann stimmt, wenn Intuitionen qua Intuitionen oder in ihrer Eigenschaft als Intuitionen für die Argumente zeitgenössischer analytischer Philosophen relevant sind. Und dass sie qua Intuitionen relevant sind, bedeutet, dass es relevant ist, dass sie Intuitionen sind und dass es ergo relevant ist, dass sie F1, F2 und F3 aufweisen. Wenn Cappelen dann aber das wirksame Vorkommen von IntuitionsMerkmalen so ausbuchstabiert, dass es impliziert, dass die Philosophinnen und Philosophen sich darüber bewusst sein (beziehungsweise annehmen) müssen, dass die Urteile, auf die sie sich berufen, F1, F2 und F3 aufweisen, dann steht dies erstens in Spannung dazu, dass Cappelen selbst immer wieder betont, dass Philosophinnen und Philosophen sich häufig über ihre eigene Vorgehensweise irren, ${ }^{32}$ und zweitens kann er dann beispielsweise daraus, dass $\mathrm{F}_{3}$ in philosophischen Argumenten nicht im von ihm spezifizierten Sinn wirksam vorkommt, nicht den oben erwähnten Schluss ziehen, dass begriffliche Wahrheiten für die Philosophie irrelevant sind: ${ }^{33}$ Dass begriffliche Wahrheiten für die Argumente der zeitgenössischen analytischen Philosophie relevant sind, würde auch dann gelten, wenn Philosophinnen und Philosophen sich regelmäßig auf begriffliche Wahrheiten berufen würden, ohne sich dessen bewusst zu sein, dass die betreffenden Urteile begrifflich wahr sind. Und mit dieser Möglichkeit setzt sich Cappelen in seiner Abhandlung nicht auseinander. Auf diese Punkte komme ich im nachfolgenden Abschnitt zurück.

\section{Das wirksame Vorkommen begrifflicher Wahrheiten}

\subsection{Indizien für die Berufung auf begriffliche Wahrheiten}

Da F1 und $F_{2}$ für das Thema des vorliegenden Buchs nicht besonders interessant sind, klammere ich diese beiden Intuitionsmerkmale für den Moment aus und gehe weiter auf Cappelens Argumente dafür ein, dass Urteile mit dem Merkmal F3, also begriffliche Wahrheiten, für die Argumente der zeitgenössischen analytischen Philosophie nicht von besonderer Bedeutung sind. Zur Vorbereitung seiner Fallstudien führt Cappelen aus, was er als Indiz dafür

$32 \quad$ Vgl. Abschnitt 4 des vorliegenden Kapitels.

33 Vgl. Cappelen 2012, S. 202. 
betrachtet, dass sich eine Philosophin oder ein Philosoph auf eine begriffliche Wahrheit abstützt:

a. Die Philosophin oder der Philosoph schreibt explizit, dass ein bestimmtes Urteil, auf das sie oder er sich beruft, begrifflich wahr ist. ${ }^{34}$

b. Die Philosophin oder der Philosoph erklärt, was sie oder er mit Begriffen meint, was es bedeutet, dass ein Urteil begrifflich wahr ist, und weshalb W. V. O. Quines und Timothy Williamsons Argumente gegen die Existenz begrifflicher Wahrheiten nicht schlüssig sind.

Da es so viele verschiedene Konzeptionen davon gibt, was Begriffe sind, und da die Auffassung, dass es begrifflich wahre Urteile gibt, derart kontrovers ist, so Cappelen, dürfen wir erwarten, dass eine Philosophin oder ein Philosoph, die oder der sich auf begriffliche Wahrheiten abstützen möchte, etwas zu den in (b) angeführten Fragen sagt. Die Abwesenheit einer entsprechenden Diskussion fasst Cappelen deshalb sogar als Hinweis darauf auf, dass die betreffende Philosophin oder der Philosoph sich nicht auf begriffliche Wahrheiten abstützt. ${ }^{35}$

c. Die Philosophin oder der Philosoph beruft sich auf ein Urteil, das F1 und F2 besitzt. $^{36}$

Den Hinweis (c) werde ich nachfolgend beiseitelassen, und zwar unter anderem deshalb, weil Cappelen selbst weiter unten schreibt, er wisse nicht, weshalb F1, F2 und $F_{3}$ sich regelmäßig gegenseitig begleiten sollten. ${ }^{37}$ Er führt diesen Hinweis lediglich an, weil seinem Verständnis nach viele Verfechterinnen und Verfechter der Auffassung, dass Intuitionen (Quellen von) philosophischer Evidenz sind, davon ausgehen, dass die drei Merkmale regelmäßig miteinander einhergehen.

d. Die Philosophin oder der Philosoph stützt sich auf ein notwendig wahres Urteil ab. ${ }^{38}$

e. Die Frage, mit der sich die Philosophin oder der Philosoph auseinandersetzt, hat mehr Ähnlichkeit mit Vixen und Tall als mit Sick und Libro:

Vixen: Consider a case in which Mark walks around in the forest and then a vixen passes right in front of him. Question: Did a female fox pass right in front of him? Tall: Consider three brothers, $A, B$, and $C$. $A$ is taller than $B . B$ is taller than $C$. Question: Is $A$ taller than $C$ ?

Sick: "A 65-year-old man is admitted with pneumonia. He has a 2-year history of Parkinson's disease and is on treatment to control his symptoms. He is an

34 Vgl. Cappelen 2012, S. 126.

35 Vgl. Cappelen 2012, S.124-127.

36 Vgl. Cappelen 2012, S. 127.

37 Vgl. Cappelen 2012, S. 128, Fußnote 14.

38 Vgl. Cappelen 2012, S. 127. 
ex-smoker of 20 cigarettes per day for 40 years. He claims he has never had a bad chest until recently but he admits this is his third attack of pneumonia in the last 6 months. Question: What is happening?" (Roper 2005, p. 131)

Libro: Mandy is walking down Russell Avenue in Buenos Aires from Thames. She walks two blocks and then turns left and walks two more blocks. Question: how many blocks away from Boutique del Libro is she? ${ }^{39}$

Cappelen schreibt, dass die Fälle, die er im Kapitel 8 seines Buches untersucht, alle eindeutig in die Kategorie von Sick und Libro gehören. ${ }^{40}$ (Die Formulierung von Sick ist dabei nicht besonders gut gewählt. »What is happening? « lässt ohne das Kontextwissen, dass Cappelen dieses Beispiel aus einem Lehrbuch für angehende Ärztinnen und Ärzte übernommen hat, vollkommen offen, worauf die Frage abzielt und um welche Art von Frage es sich mithin handelt. »Was passiert?« könnte beispielsweise auch mit einer Wiederholung der präsentierten Schilderung respektive mit einer Paraphrase der gegebenen Beschreibung beantwortet werden, und in diesem Fall würde die Antwort wie im Falle von Vixen und Tall begrifflich aus der Fallbeschreibung folgen.)

Die Argumente, die Cappelen im Zusammenhang mit den einzelnen Fallstudien dafür anführt, dass die jeweils relevanten Urteile nicht die Eigenschaft $\mathrm{F}_{3}$ aufweisen beziehungsweise dass die jeweils relevanten Argumente nicht auf begriffliche Wahrheiten abgestützt sind, passen zur obigen Liste von Indizien:

(i) Die Autorin oder der Autor bespricht nicht die Fragen, was Begriffe sind, was es für ein Urteil bedeutet, begrifflich wahr zu sein, usw. ${ }^{41}$

(ii) Die Autorin oder der Autor betont an keiner Stelle, dass irgendein Urteil, auf das sie oder er sich beruft, notwendig wahr sei. ${ }^{42}$

(iii) Die Autorin oder der Autor macht nicht den Eindruck, sie oder er beabsichtige, sich ausschließlich auf ihre oder seine Begriffskompetenz abzustützen. ${ }^{43}$

(iv) Die Autorin oder der Autor glaubt nicht an die Existenz begrifflich wahrer Urteile. ${ }^{44}$

(v) Die Autorin oder der Autor macht nicht den Eindruck, als würde sie oder er notwendige, begrifflich wahre oder $A$ priori-Urteile als philosophisch interessanter betrachten als andere Urteile. ${ }^{45}$

\footnotetext{
39 Cappelen 2012, S. 127.

40 Vgl. Cappelen 2012, S. $127 \mathrm{f}$.

41 Vgl. Cappelen 2012, S. 166, 168, 173.

42 Vgl. Cappelen 2012, S. $146 f$.

43 Vgl. Cappelen 2012, S. 136f., 146, 153, 161, 166, 168, 179.

44 Vgl. Cappelen 2012, S. 146, 147, 178.

45 Vgl. Cappelen 2012, S. 146, 179.
} 
(vi) Die Negation des relevanten Urteils bringt eine Möglichkeit zum Ausdruck. ${ }^{46}$

(vii) Das relevante Urteil wird unter anderem auf der Grundlage empirischer Daten oder auf der Basis von Ergebnissen empirischer Wissenschaften gefällt. ${ }^{47}$

(viii) Das Argument funktioniert ohne die Annahme, eines der involvierten Urteile sei begrifflich wahr. ${ }^{48}$

(ix) (F1 und F2 fehlen. ${ }^{49}$ )

Die Argumente (i) bis (v) sprechen allerdings höchstens dagegen, dass eine Philosophin oder ein Philosoph sich wissentlich auf begriffliche Wahrheiten beruft respektive dass sie oder er beabsichtigt, sich auf begriffliche Wahrheiten zu berufen. ${ }^{50}$ Wie bereits weiter oben erwähnt, besteht jedoch auch die Möglichkeit, dass Philosophinnen und Philosophen sich regelmäßig auf begriffliche Wahrheiten berufen, ohne sich dieses Umstands beziehungsweise dieser Eigenschaft der betreffenden Urteile bewusst zu sein. Im Zusammenhang mit den weiter unten besprochenen Beispielen wird sich herausstellen, dass diese Hypothese tatsächlich eine gewisse Plausibilität hat. Und sollte es tatsächlich so sein, dass Philosophinnen und Philosophen sich häufig unbewusst auf begriffliche Wahrheiten berufen, wäre dies offensichtlich relevant für Cappelens Untersuchung. In Philosophy without Intuitions finden wir nämlich beispielsweise die folgende Behauptung: »In the case studies that I investigate in [chapter 8], we will consistently find an absence of all of $\mathrm{F}_{1}-\mathrm{F}_{3}{ }{ }^{5}{ }^{51}$ Und um zu einer solchen Behauptung berechtigt zu sein, müsste Cappelen natürlich auch eine Berufung auf $\mathrm{F}_{3}$ (und auf die anderen Merkmale) ausschließen können, die von den betreffenden Philosophinnen und Philosophen unbemerkt bleibt.

Wie bereits angemerkt, äußert sich Cappelen in anderen Zusammenhängen skeptisch dazu, wie ernst wir die Charakterisierungen nehmen sollten, die Philosophinnen und Philosophen von ihrer eigenen Vorgehensweise liefern. Am deutlichsten formuliert er diese Bedenken in der folgenden Passage:

\footnotetext{
46 Vgl. Cappelen 2012, S. 138, 182.

47 Vgl. Cappelen 2012, S. 135-137, 152-154, 179.

48 Vgl. Cappelen 2012, S. $186 f$.

49 Vgl. Cappelen 2012, S. 145, 178.

5o (i) beruht außerdem auf unangemessen hohen Erwartungen, und (v) ist als Anhaltspunkt inadäquat, da nicht klar ist, weshalb ein Philosoph oder eine Philosophin, der oder die sich auf Intuitionen als (Quelle von) Evidenz beruft, deswegen andere Urteile als philosophisch weniger relevant auffassen sollte.

51 Cappelen 2012, S. 114.
} 
Being good at thinking about trolley cases doesn't imply that you are good at thinking what you do when you think about trolley cases - no more so than being a good mathematician implies being good at thinking about the nature of mathematical reasoning. As a result we sometimes find authors who make excellent contributions to a topic saying false things about how they go about making those contributions. As I emphasized in Chapter 1, endorsement of Centrality is part of many analytic philosophers' self-conception. Since Centrality is false, many philosophers will say false things and have false beliefs about what they do when they philosophize, which is no more incompatible with being an excellent philosopher than being confused about metamathematics is incompatible with being an excellent mathematician. ${ }^{52}$

Im Lichte solcher Äußerungen ist es erstaunlich, dass Cappelen, wenn es um die Frage geht, ob Philosophinnen und Philosophen sich auf Urteile mit den Eigenschaften $\mathrm{F}_{1}$ bis $\mathrm{F}_{3}$ berufen, die methodologischen Selbsteinschätzungen der besprochenen Autorinnen und Autoren plötzlich für bare Münze zu nehmen scheint.

Interessanter als die Argumente (i) bis (v) sind deswegen die Argumente (vi) bis (viii). In den nächsten drei Abschnitten werde ich allerdings dafür argumentieren, dass es auch im Falle der Argumente (vi) bis (viii) jeweils entweder so ist, dass sie für die von Cappelen diskutierten Fälle nicht einschlägig sind, oder dass sie schon an und für sich als Argumente gegen das Vorliegen einer Berufung auf eine begriffliche Wahrheit ungeeignet sind.

\subsection{Argument (vi): Konsistenz der Negation}

\subsubsection{Perrys Supermarkt-Fall I}

Wenden wir uns zunächst dem Argument (vi) zu. Dass die Negation eines bestimmten Urteils, auf das sich eine Philosophin oder ein Philosoph beruft, eine Möglichkeit zum Ausdruck bringt, zieht Cappelen zweimal als Argument dafür heran, dass das betreffende Urteil nicht begrifflich wahr ist. Das erste Mal im Zusammenhang mit John Perrys Supermarkt-Beispiel, das zusammengefasst wie folgt lautet: Perry ist mit einem Einkaufswagen im Supermarkt unterwegs und bemerkt am Boden eine Zucker-Spur. Er folgt der Spur, um dem dafür verantwortlichen Kunden oder der Kundin mitzuteilen, dass dieser oder diese mit einer kaputten Zucker-Packung unterwegs ist. Nach einigen Runden um ein großes Gestell herum scheint er den Verursacher oder die Verursacherin

52 Cappelen 2012, S. 163. Vgl. auch S. 206: »Just as philosophers in moments of confused meta-reflection will often describe what they do as relying on intuitions, they will often mistakenly describe what they do as 'analysis', but this turns out to be yet another instance of the familiar phenomenon of someone engaging in an activity he or she is not particularly good at giving a description of.« 
der Verunreinigung immer noch nicht eingeholt zu haben, aber die ZuckerSpur wird immer dicker. Da dämmert es ihm, dass der Kunde mit der defekten Zucker-Packung er selbst ist. Er hört deshalb auf, der Spur zu folgen, und kümmert sich stattdessen um die kaputte Packung in seinem Einkaufswagen. ${ }^{53}$

Perry geht es in seinem Artikel darum, die Proposition zu identifizieren, die er zum Schluss des Beispiels anfängt zu glauben und unter Rückgriff auf welche die Veränderung in seinem Verhalten erklärt werden kann. Der Satz »Ich habe eine kaputte Zucker-Packung im Wagen« scheint keine spezifische Proposition zu bestimmen, da dieser Satz je nach Sprecherin oder Sprecher unterschiedliche Propositionen ausdrückt. Wenn wir aber »ich« durch einen anderen Ausdruck ersetzen, zum Beispiel durch »John Perry«, dann können wir damit nicht Perrys Verhalten erklären, ohne ihm zusätzlich die Überzeugungen »Ich bin John Perry« zuzuschreiben, womit wir wieder bei einem Satz angelangt wären, der den indexikalischen Ausdruck »ich« enthält und je nach Sprecherin oder Sprecher unterschiedliche Propositionen ausdrückt. Außerdem sind wir mit dem Problem konfrontiert, dass Perry sowohl vor als auch nach der Entdeckung, dass er selbst der Kunde mit der defekten ZuckerPackung ist, das folgende Urteil glaubt: »Die Person, die eine Zucker-Spur im Supermarkt hinterlässt, hat eine kaputte Zucker-Packung im Wagen«. Und da John Perry selbst die Person ist, die eine Zucker-Spur im Supermarkt hinterlässt, handelt diese Überzeugung auch bereits vor Perrys Entdeckung, dass er ebendiese Person ist, von ihm selbst. Man könnte deshalb auch auf die Idee kommen, dass die Veränderung in Perrys Verhalten gar nicht mit Bezug auf eine bestimmte Proposition erklärt werden kann, die Perry zunächst nicht glaubt, aber später schon.

In Cappelens Diskussion der Frage, ob in der Debatte um Perrys Gedankenexperiment auf irgendwelche Urteile, die F1, F2 oder F3 aufweisen, zurückgegriffen wird, zieht Cappelen unter anderem $\mathrm{P}_{1}$ in Erwägung:

P1: Some cognitive change happened to Perry and that change figures in an explanation of his change in behavior. ${ }^{54}$

Es ist dieses Urteil, über das Cappelen später behauptet, seine Negation sei möglich und das Urteil sei somit nicht begrifflich wahr:

53 Vgl. Cappelen 2012, S. 133, Perry 1979, S. 3.

54 Cappelen 2012, S. 137. 
Can an argument be made that $\mathrm{P}_{1}$ is conceptually justified? [...] Conceptually justified propositions are, according to the views surveyed in Chapter 6, necessary truths. P1 is not. It is possible for the kind of change in behavior to happen without a cognitive change. ${ }^{55}$

Cappelen machtallerdings eine Seite weitervorne selbst geltend, dass Pije nachdem, wie wir »cognitive« auffassen, implizit bereits Teil der Fallbeschreibung sei und deshalb keine interessante Erkenntnis zum Ausdruck bringe. ${ }^{56} \mathrm{Er}$ erklärt aber nicht, unter welcher Interpretation von »cognitive« dies nicht der Fall sei. Im Gegensatz zu Cappelen sehe ich nicht, dass es eine Bedeutung von »cognitive« gibt, der entsprechend man P1 so verstehen könnte, dass dieses Urteil nicht von Perrys Fallbeschreibung impliziert wird. Deshalb glaube ich, dass Cappelen falsch liegt mit der Behauptung, die Negation von P1 sei möglich. Es mag vielleicht - abstrahiert von der restlichen Fallbeschreibung - möglich sein, dass Perry sich auf die beschriebene Weise verhält, ohne dass sich kognitiv bei ihm etwas verändert (wobei interessant wäre, was Cappelen mit »möglich « meint. Begrifflich möglich? Nomologisch möglich?). Aber wenn wir die Fallbeschreibung voraussetzen (was ja bei Fragen, die sich auf Gedankenexperimente beziehen, die Idee ist), kann sich P1 meines Erachtens nicht als falsch herausstellen.

\subsubsection{Williams' Folter-Fall}

Kommen wir nun zum zweiten Fall, in dem Cappelen über ein für ein philosophisches Argument relevantes Urteil sagt, es könne nicht begrifflich wahr sein, da seine Negation eine Möglichkeit ausdrücke. Das betreffende Urteil kommt im Zusammenhang mit Bernard Williams' Folter-Beispiel zur Sprache. Dieses Beispiel wird von Williams in Verbindung mit der Idee besprochen, dass personale Identität an Erinnerungen geknüpft ist, und dass jeder und jede von uns, grob gesagt, diejenige Person ist, deren Erinnerungen er oder sie hat. Williams diskutiert in diesem Kontext den folgenden Fall: Wenn ich erfahre, dass ich morgen gefoltert werde, wird mich diese Erwartung mit Furcht erfüllen, und diese Furcht würde auch dadurch nicht gelindert werden, dass mir versprochen wird, dass ich vor meiner Folter einer Operation unterzogen werde, die zur Folge hat, dass ich sämtliche Erinnerungen, über die ich jetzt verfüge, verliere und an ihrer Stelle die Erinnerungen einer anderen Person, die

55 Cappelen 2012, S. 138, Kursivierung im Original.

56 Vgl. Cappelen 2012, S. 137. 
jetzt auch lebt, »in mein Gehirn kopiert« werden. ${ }^{57}$ Das Urteil, das Cappelen in Bezug auf dieses Beispiel diskutiert, ist das folgende:

P2: Die Mitteilung, dass ich vor der Folter der beschriebenen Operation unterzogen werde, würde meine Furcht nicht lindern.

Dieses Urteil interpretiert Cappelen als Hypothese darüber, wie ich emotional in einer bestimmten Situation reagieren würde. Das Urteil, so Cappelen, weist nicht die Eigenschaft $\mathrm{F}_{3}$ auf, was man unter anderem daran sieht, dass seine Negation eine Möglichkeit ausdrückt: Ein Szenario, in welchem die Mitteilung, dass ich zum Zeitpunkt der Folter nicht mehr meine Erinnerungen, sondern die Erinnerungen einer anderen Person haben werde, dazu führt, dass ich mich beruhige, ist nicht inkonsistent. ${ }^{58}$

In Williams' Beschreibung des Beispiels wird dem zu Folternden die oben genannte Mitteilung in mehreren Schritten gemacht: Du wirst dich zum Zeitpunkt der Folter nicht mehr an diese Ankündigung erinnern «, »Du wirst sogar alle deine Erinnerungen verloren haben «, »Stattdessen wirst du andere Erinnerungen haben «, usw., und nach jedem Schritt merkt Williams an, dass ihn dies nicht beruhigen würde, dass er immer noch Angst hätte etc. Nach dem allerletzten Schritt der Ankündigung heißt es dann aber leicht verändert: Fear, surely, would still be the proper reaction. ${ }^{59}$ Diese letzte Aussage definitiv keine Hypothese darüber, welche emotionale Reaktion Williams auf kontrafaktische Ereignisse hätte, sondern eine Behauptung über die Angemessenheit einer bestimmten emotionalen Reaktion. Und ich glaube, dass es Williams von Anfang um diese Angemessenheits-Frage geht. Ob es angemessen ist, dass ich mich nach der Ankündigung, dass ich zum Zeitpunkt der Folter nicht mehr meine eigenen, sondern fremde Erinnerungen habe, weiterhin fürchte, hängt nämlich offensichtlich eng mit der Frage zusammen, ob die Person mit den fremden Erinnerungen dann noch ich wäre. ${ }^{60}$ Demgegenüber ist die Idee, dass uns der kontingente Umstand, dass jemand eine bestimmte emotionale

57 Vgl. Williams 1970, S. 167f. Die etwas problematische Formulierung »in mein Gehirn kopiert« (»copied into my brain«) habe ich von Williams übernommen.

58 Vgl. Cappelen 2012, S. 182.

59 Williams 1970, S. 168.

6o Und diese letzte Frage dürfte eine begriffliche Frage sein - was nicht bedeutet, dass sie auf der Basis einer Begriffsanalyse eindeutig mit Ja oder Nein beantwortet werden kann. Es erscheint mir plausibel, dass unser Begriff der Person nicht spezifiziert ist für den Fall von Wesen, deren Erinnerungen den Körper wechseln können. Aber relevant für die Beantwortung (oder Zurückweisung) der Frage werden einzig begriffliche Zusammenhänge sein. 
Reaktion zeigt, darüber Aufschluss geben sollte, wie es um Verhältnisse der personalen Identität bestellt ist, offensichtlich abwegig.

Wir könnten also zum Schluss kommen, dass für Williams eigentlich nicht $\mathrm{P}_{2}$, sondern $\mathrm{P}_{3}$ relevant ist:

$\mathrm{P}_{3}$ : Nach der Mitteilung, dass ich vor der Folter der beschriebenen Operation unterzogen werde, wäre Furcht nach wie vor eine adäquate Reaktion.

Und um zu zeigen, dass sowohl $\mathrm{P}_{3}$ als auch die Negation davon unter den im Gedankenexperiment beschriebenen Bedingungen eine Möglichkeit zum Ausdruck bringen, scheint mir - anders als im Fall von P2 - wenigstens ein gewisser Argumentationsaufwand geleistet werden zu müssen.

\subsection{Argument (vii): Begründung mittels empirischer Daten}

\subsubsection{Perrys Supermarkt-Fall II}

Wie bereits erwähnt, führt Cappelen als Argument dafür, dass bestimmte philosophisch relevante Urteile nicht begrifflich wahr sind, manchmal auch an, dass diese Urteile auf der Grundlage empirischer Daten oder auf der Basis der Ergebnisse empirischer Wissenschaften gefällt werden. Dieses Argument führt Cappelen unter anderem im Zusammenhang mit dem bereits unter 3.2.1 besprochenen Supermarkt-Fall von John Perry an. Dieser Fall wirft Cappelen zufolge eine ganze Reihe von Fragen auf, zu denen unter anderem die folgenden gehören:

[W] hat is the connection between change in belief state and the content of a belief? Can a change in content of belief explain Perry's change in behavior? What is the content of a first-person belief? How do we articulate the change in content - i.e. how do we, who are not that person and so cannot use the firstperson pronoun, articulate the change? ${ }^{61}$

Die Philosophinnen und Philosophen, die sich seit dem Erscheinen von Perrys Artikel an der Debatte um diese Probleme beteiligt haben, so Cappelen, haben sich dabei unter anderem auf Resultate der Linguistik und der Psychologie berufen, und auf »contingent features of human cognition « ${ }^{62}$ Mir ist allerdings nicht ersichtlich, wie empirische Befunde im Rahmen der Befassung mit Perrys Supermarkt-Fall relevant werden könnten. Wenn wir Perrys eigenen Artikel und seine Beantwortung der Ausgangsfrage desselben betrachten, wird darin jedenfalls nicht auf empirische Daten Bezug genommen, weder auf solche der

61 Cappelen 2012, S. 134.

62 Vgl. Cappelen 2012, S. 136. 
Linguistik noch auf solche der Psychologie. In einem der Artikel, die Cappelen in diesem Zusammenhang anführt, findet demgegenüber tatsächlich eine Berufung auf empirische Entdeckungen statt, und zwar in Dilip Ninans »De Se Attitudes « (2010). Unter anderem macht Ninan geltend, dass die Übersetzung des Satzes »John sagte, dass ich ein Held sei« ins Amharische ambig sei zwischen unserem $» J o h n$ sagte, dass ich (also die Sprecherin oder der Sprecher dieses Satzes) ein Held sei« und $» J o h n$ sagte über sich selbst, dass er ein Held sei« ${ }^{63}$ Dieser empirische Befund ist für Ninan aber nur deshalb relevant, weil er sich mit der Analyse von Propositionen, die von der Sprecherin oder dem Sprecher selbst handeln, in einer ganz bestimmten formalen Sprache befasst und offenbar eine Analyse sucht, die zu allen natürlichen Sprachen passt und auch zu Fällen, in welchen solche Propositionen in Nebensätzen zum Ausdruck gebracht werden. Dies ist offensichtlich ein ganz anderes Projekt als das von Perry, und zwar ein teilweise empirisch-linguistisches Projekt. Cappelen bleibt den Nachweis dafür schuldig, dass es Philosophinnen oder Philosophen gibt, die sich im Rahmen der Beantwortung von Perrys Fragen auf empirische, linguistische Befunde berufen haben.

Auf Ergebnisse der Psychologie nimmt Ninan keinen Bezug, und dasselbe gilt, soweit ich sehe, auch für Robert Stalnakers Besprechung des Themas »selflocating belief «, die Cappelen in diesem Kontext ebenfalls anführt. ${ }^{64}$ Wenn wir die oben zitierte Auswahl von Fragen betrachten, könnten wir eventuell auf die Idee kommen, es sei eine psychologische Frage, welche kognitiven Veränderungen die Veränderung von Perrys Verhalten erklären können. Zumindest dann, wenn wir »Erklären« in Abgrenzung zu »Begründen« zur Bezeichnung von Kausal-Erklärungen auffassen. Wir müssten dann testen, ob das Erlangen der Überzeugung, eine kaputte Zuckerpackung im Einkaufswagen zu haben, tatsächlich in einem statistisch relevanten Verhältnis zum Verhalten, sich in seinen Wagen zu bücken, steht.

Darauf, wie Cappelens Frage »Can a change in content of belief explain Perry's change of behavior?« in ihrem gewöhnlichen Sinn zu beantworten ist, haben Experimente der im vorangehenden Absatz angedeuteten Art aber keinen Einfluss. Natürlich ist es prinzipiell möglich, dass wir herausfinden, dass eine überwiegende Mehrheit von Personen, die die Überzeugung erlangen, eine kaputte Zuckerpackung im Wagen zu haben, sich nicht in ihren Wagen bückt, um das Problem zu beheben (wobei mich das überraschen würde). Aber dieser Befund würde in keiner Weise nahelegen, dass wir Perrys im Gedankenexperiment beschriebenes Verhalten nicht damit erklären können, dass er die

63 Vgl. Ninan 2010, S. 556.

64 Vgl. Stalnaker 2008, Kap. 3. 
Überzeugung erlangt hat, dass er eine kaputte Zuckerpackung im Wagen hat. Denn dass das Erlangen dieser Überzeugung als Erklärung für dieses Verhalten fungieren kann, ist eine begriffliche, keine empirische Wahrheit. ${ }^{65}$

\subsubsection{Thomsons Violinisten-Fall}

Der zweite Kontext, in welchem Cappelen über ein für ein philosophisches Argument relevantes Urteil sagt, es beruhe auf empirischen Tatsachen, ist Judith Jarvis Thomsons Violinisten-Beispiel. Dieses Beispiel dient dazu, bestimmte Argumente zu entkräften, die gegen das Recht auf Abtreibung vorgebracht worden sind und grob wie folgt funktionieren: Zwar hat jede Person grundsätzlich ein Recht auf die Entscheidung darüber, was mit ihrem Körper passiert, aber das Recht auf Leben einer Person $A$ überwiegt im Zweifelsfall das Selbstbestimmungsrecht einer anderen Person $B$. Angenommen, dass ungeborene Kinder Personen sind, ist Abtreibung also nicht erlaubt. ${ }^{66}$ Thomson präsentiert den Fall, in welchem eine Person $S$ eines Morgens im Spital erwacht und an das Blutkreislaufsystem eines berühmten Violinisten angeschlossen ist, der an einer schweren Nierenkrankheit leidet und nicht überlebt hätte, wenn $S$ nicht entführt und mit dem Violinisten verbunden worden wäre. Würde man $S$ und den Violinisten vor dem Ablauf von neun Monaten trennen, würde der Violinist sterben. ${ }^{67}$ Wenn wir in diesem Fall nicht der Auffassung sind, dass $S$ moralisch dazu verpflichtet ist, sich an den Violinisten angeschlossen zu lassen und neun Monate lang mit ihm im Spitalbett zu liegen, dann kann das oben skizzierte Anti-Abtreibungs-Argument auch nicht schlüssig sein. ${ }^{68}$

Im Anschluss an seine Rekonstruktion von Thomsons Beispiel argumentiert Cappelen unter anderem dafür, dass das Urteil $\mathrm{P}_{4}$ keine begriffliche Wahrheit sei:

P4: $S$ ist nicht moralisch dazu verpflichtet, neun Monate lang an den Violinisten angeschlossen zu bleiben. ${ }^{69}$

An dieser Stelle sollte zunächst einmal betont werden, dass Moralphilosophinnen und -philosophen, die ihre Arbeit so auffassen, dass sie auf Intuitionen abgestützt ist, üblicherweise nicht davon ausgehen, dass moralische

$65 \mathrm{Ob}$ »erklären« dabei sinnvollerweise kausal aufgefasst werden kann, ist umstritten, und ich möchte mich im Rahmen der vorliegenden Abhandlung auf keine der beiden Seiten der Debatte schlagen.

66 Vgl. Cappelen 2012, S. 149, Jarvis Thomson 1971, S. 48.

67 Vgl. Cappelen 2012, S. 149, Jarvis Thomson 1971, S. $48 \mathrm{f}$.

68 Vgl. Cappelen 2012, S. 150, Jarvis Thomson 1971, S. 49.

69 Vgl. Cappelen 2012, S. 153f. 
Intuitionen das Merkmal $\mathrm{F}_{3}$ aufweisen, d.h. ausschließlich auf Begriffskompetenz beruhen. Indem Cappelen für die Identifikation von Intuitionen auch in der Besprechung moralphilosophischer Argumente am Merkmal F3 festhält, wendet er sich also gewissermaßen gegen einen Strohmann.

Ich selbst bin ebenfalls nicht der Auffassung, dass moralische Urteile im Allgemeinen und $\mathrm{P}_{4}$ im Besonderen begrifflich wahr sind, möchte aber dennoch gegen die Stichhaltigkeit von Cappelens Argument dagegen, dass $\mathrm{P}_{4}$ begrifflich wahr ist, argumentieren. Cappelen meint, beim Fällen dieses Urteils würden wir uns unter anderem auf kontingente Tatsachen über menschliche Leben und Vorlieben berufen, nämlich unter anderem darauf, dass Menschen es schätzen, dazu in der Lage zu sein, sich unabhängig von anderen Personen zu bewegen, und dass die Lebensqualität einer Person herabgesetzt wird, wenn sie dazu gezwungen ist, neun Monate im Bett zu verbringen. ${ }^{70}$ Diese beiden Urteile scheinen mir jedoch Selbstverständlichkeiten zu sein von der Art, wie sie von den meisten Argumenten vorausgesetzt werden. Betrachten wir als Beispiel noch einmal den folgenden Fall aus der Epistemologie:

Bob has a friend, Jill, who has driven a Buick for many years. Bob therefore thinks that Jill drives an American car. He is not aware, however, that her Buick has recently been stolen, and he is also not aware that Jill has replaced it with a Pontiac, which is a different kind of American car. Does Bob really know that Jill drives an American car $[\ldots]$ ? $^{71}$

Dass die meisten Leute geneigt sind, diese Frage zu verneinen, hängt unter anderem an der kontingenten Tatsache, dass es nicht der Fall ist, dass (jeder weiß, dass) es nur amerikanische Automarken gibt. Das zeigt aber nicht, dass wir nicht sagen sollten, dass das Urteil, dass Bob nicht weiß, dass Jill einen amerikanischen Wagen fährt, begrifflich aus der obigen Fallbeschreibung folgt. Es besteht kein Bedürfnis, in philosophischen Fallbeschreibungen auf Selbstverständlichkeiten hinzuweisen, die sämtlichen potentiellen Leserinnen und Lesern ohnehin bewusst sind.

Cappelen bespricht einen ähnlichen Einwand wie den gerade von mir vorgebrachten im Zusammenhang mit einem anderen Fallbeispiel. Nämlich den Einwand, dass Informationen wie die oben genannten (Menschen bevorzugen es, über Bewegungsfreiheit zu verfügen, und verbringen ungern neun Monate im Bett), implizit Teil der zu beurteilenden Szenarien sind. Cappelens Replik auf diesen Einwand besteht darin, dass wir aus jeder empirischen Frage eine begriffliche Frage machen können, indem wir die

$70 \quad$ Vgl. Cappelen 2012, S. 153 f.

71 Weinberg/Nichols/Stich 2001, S. 443. 
Frage mit genügend Zusatzinformationen anreichern. Im oben erwähnten Libro-Beispiel (S. 227 des vorl. Kapitels) können wir die Fallbeschreibung zum Beispiel durch eine Beschreibung des relevanten Abschnitts des Stadtplans von Buenos Aires ergänzen, und dann folgt die richtige Antwort auf die Frage, wie viele Blocks Mandy von der Boutique del Libro entfernt ist, begrifflich aus der Fallbeschreibung. ${ }^{72}$ Aber die Aufgabe, vor die uns die ursprüngliche Libro-Beschreibung stellt, ist, so Cappelen, offensichtlich verschieden von der Aufgabe, vor die uns ein durch diese ganzen geographischen Informationen über Buenos Aires ergänzter Libro-Fall stellt: Wir haben es im entscheidenden Sinn eben nicht mehr mit demselben Fall zu tun. Und die Möglichkeit, einen Fall so zu ergänzen, dass seine Antwort begrifflich aus der Fragestellung folgt, zeigt selbstverständlich nicht, dass auch der nicht ergänzte Fall auf die Beantwortung einer begrifflichen Frage abstellt. ${ }^{73}$

Meines Erachtens verhalten sich der Violinisten-Fall und der Fall um Bob und Jills Auto allerdings nicht analog zum Libro-Fall: Während für die Beantwortung der Fragen im Violinisten- und im Bob-Fall nur solche empirischen Tatsachen vorausgesetzt werden müssen, deren Bestehen für praktisch jeden erwachsenen Menschen selbstverständlich ist, müssen im Libro-Fall Informationen ergänzt werden, die nicht allgemein bekannt sind.

\subsubsection{Cappelens und Hawthornes Chicha mascada-Fall}

Ich komme nun zum dritten und letzten Fall, in dem Cappelen über ein für ein philosophisches Argument relevantes Urteil sagt, es beruhe auf empirischen Tatsachen und könne deshalb nicht begrifflich wahr sein. Das betreffende Argument stammt aus Relativism and Monadic Truth von John Hawthorne und Cappelen selbst. In der relevanten Passage setzen sich die beiden Autoren mit der Frage auseinander, ob zwei Äußerungen, »Chicha mascada ist köstlich « ${ }^{74}$ und »Chicha mascada ist widerlich« (bzw. auch »Chicha mascada ist nicht köstlich«), getätigt von zwei verschiedenen Personen, in einem Widerspruch zueinander stehen. Cappelen und Hawthorne verteidigen die Auffassung, dass sich das spontane Urteil vieler Personen, dem zufolge die beiden Äußerungen sich in der Tat widersprechen, bei näherer Betrachtung als falsch herausstellt. Dabei argumentieren sie unter anderem wie folgt: Wenn wir uns vergegenwärtigen, in welchem Maß unsere Geschmacksurteile von kontingenten

72 Cappelen drückt sich hier natürlich etwas vorsichtiger aus, da er die Auffassung ablehnt, dass es begriffliche Wahrheiten gibt.

73 Vgl. Cappelen 2012, S. 156-158.

74 Chicha mascada (von Cappelen irrtümlich »chica mascada« geschrieben) ist ein Getränk, das unter anderem aus von verschiedenen Personen zerkauten und in eine Schüssel gespuckten Maiskörnern hergestellt wird. Vgl. Stübe 1918, S. 59. 
Faktoren abhängen und welchen Veränderungen sie im Laufe unseres Lebens unterzogen waren, verlieren wir das Gefühl, dass fremde Geschmacksurteile mit unseren eigenen Urteilen im Widerspruch stehen. Cappelen und Hawthorne vertreten also die These $\mathrm{P}_{5}$ :

P5: Die beiden Äußerungen »Chicha mascada ist köstlich« und »Chicha mascada ist widerlich «, getätigt von verschiedenen Sprecherinnen oder Sprechern, bringen keine miteinander inkonsistenten Urteile zum Ausdruck.

Dieses Urteil weist Cappelen zufolge nicht das Merkmal F3 auf: Die beiden Autoren haben sich beim Fällen des Urteils nicht ausschließlich auf ihre Begriffskompetenz abgestützt, sondern auch auf Erkenntnisse der empirischen Wissenschaften, unter anderem der Anthropologie:

Is it plausible, on any construal of 'concept', 'conceptual competence' and 'justified', that [Cappelen and Hawthorne's] claim that the two imagined utterances of 'Chica mascada is delicious' and 'Chica mascada is disgusting' don't express disagreement, is justified simply by their competence with disagreement, delicious, disgusting and chica mascada? As we have seen, it is hard to find proof for or against any such hypothesis given the vague and variable characterizations of $F_{3}$, but, speaking now as one of the co-authors, I can report that the work that led up to those claims did not resemble anything that we know as being described as conceptual analysis: we read anthropology books about disgust reactions in various cultures, we tried to learn a bit about the cultural variations in disgust reactions, their various physical expressions, and the various hypotheses about the evolutionary source of disgust reactions. It would just be silly to insist that this entire process should be characterized as 'relying solely on our conceptual competence. ${ }^{75}$

Nun möchte ich Cappelen und Hawthorne natürlich nicht absprechen, dass sie sich im Zuge ihrer Erwägungen darüber informiert haben, wie Ekel in verschiedenen Kulturen ausgedrückt wird, wie die Existenz von Ekelreaktionen evolutionär begründet werden kann und so weiter. Ich bin auch bereit einzuräumen, dass $\mathrm{F}_{3}$ in der Argumentation von Cappelen und Hawthorne kein wirksames Vorkommen im von Cappelen bestimmten Sinn hat. Und natürlich will man den ganzen Prozess, den Cappelen und Hawthorne durchlaufen haben, nicht als »sich ausschließlich auf seine Sprachkompetenz abstützen « charakterisieren, aber wie man diesen ganzen Prozess beschreiben würde, steht ja schließlich auch nicht zur Debatte.

75 Cappelen 2012, S. 178f., Kursivierung im Original. 
Wir haben weiter oben bereits gesehen, dass es für die ambitionierten Schlussfolgerungen, die Cappelen in seinem Buch zieht, nicht ausreichend ist, festzustellen, dass $\mathrm{F}_{3}$ in den von ihm besprochenen Argumenten kein wirksames Vorkommen hat, sondern dass es für sein Unterfangen auch interessant ist, ob eine begriffliche Wahrheit in den Argumenten von Philosophinnen und Philosophen eine zentrale Rolle spielt, die sich nicht darüber bewusst sind, dass das betreffende Urteil begrifflich wahr ist. Und genau mit einem solchen Fall haben wir es hier zu tun. Denn $\mathrm{P}_{5}$ ist definitiv entweder begrifflich wahr oder begrifflich falsch. Ob ein Sprecher, der sagt: Chicha mascada ist köstlich«, einer Sprecherin widerspricht, die sagt: »Chicha mascada ist widerlich «, hängt einzig und allein von der Bedeutung solcher Äußerungen ab, und um die Frage zu beantworten, ob $\mathrm{P}_{5}$ wahr oder falsch ist, müssen wir also auf nichts anderes zurückgreifen als auf unsere Sprach- oder Begriffskompetenz. Das bedeutet nicht, dass die Beantwortung dieser Frage ein triviales Unterfangen ist. Wir können kompetente Verwenderinnen oder Verwender der Begriffe »köstlich « und »widerlich « sein, ohne spontan dazu in der Lage zu sein, das Verhältnis zu bestimmen, in dem die Geschmacksurteile verschiedener Sprecherinnen und Sprecher zueinander stehen. Aber Analoges gilt auch für P6, ohne dass es in diesem Fall angemessen wäre, daran zu zweifeln, dass es sich dabei um eine begriffliche Wahrheit oder Falschheit handelt:

P6: Die Äußerung »Mein Vater ist Chirurg«, getätigt von Sprecher A, steht im Widerspruch zur Äußerung »In meiner Familie väterlicherseits arbeitet niemand im Gesundheitssektor«, getätigt von der Adoptivtochter von Sprecher A.

Auch hier könnte natürlich jemand gelten machen, er habe sich zur Beantwortung der Frage, ob dieses Urteil wahr oder falsch sei, mit der Verbreitung von Ärzten in Familien, die Kinder adoptieren, befasst oder mit der Wahrscheinlichkeit, dass von jemandes Familie väterlicherseits mindestens eine Person im Gesundheitswesen tätig ist, aber trotzdem haben die Ergebnisse entsprechender Studien keinen Einfluss darauf, wie die Wahrheit von P6 zu beurteilen ist. Und ebenso verhält es sich auch bei dem Problem, mit dem sich Cappelen und Hawthorne auseinandersetzen: Wie Ekel in verschiedenen Kulturen zum Ausdruck gebracht wird und evolutionär begründbar ist, ist irrelevant für die Frage, ob $\mathrm{P}_{5}$ wahr oder falsch ist. 


\subsection{Argument (viii): Entbehrlichkeit der Einschätzung eines Urteils als begrifflich wahr}

Damit kommen wir auch schon zum letzten Argument, das Cappelen in seinen Fallstudien dafür anführt, dass in einem bestimmten von ihm untersuchten Fall keine Abstützung auf ein F3-Urteil stattfindet:

(viii) Das Argument funktioniert ohne die Annahme, eines der involvierten Urteile sei begrifflich wahr.

Dieses Urteil fällt Cappelen in Bezug auf Chalmers' Zombie-Argument gegen den Materialismus, das Chalmers selbst wie folgt zusammenfasst: Eine Welt, die physikalisch identisch mit unserer Welt ist, in der es aber keine Wesen mit Bewusstsein gibt, ist vorstellbar (eigentlich: »ideally primarily positively (negatively) conceivable«). Also ist eine solche Welt möglich (eigentlich: "primarily possible«). Also ist der Materialismus falsch. ${ }^{76}$ Die detaillierte Ausbuchstabierung von Chalmers' Argument und seiner Auffassung der relevanten Vorstellbarkeits- und Möglichkeitsbegriffe braucht uns hier nicht zu interessieren. Cappelen befasst sich jedenfalls mit der Frage, ob Chalmers' Urteil, eine Zombie-Welt sei vorstellbar, F3 aufweise. Er äußert die Befürchtung, dass Chalmers selbst dieses Urteil als begriffliche Wahrheit auffasst, macht aber Folgendes geltend: Viele Philosophinnen und Philosophen, die das Zombie-Argument überzeugend finden, sind entweder agnostisch in Bezug auf die Frage, ob Vorstellbarkeits-Urteile begriffliche Wahrheiten oder Falschheiten sind, oder verneinen diese Frage. Die Auffassung, dass das Urteil, eine Zombie-Welt sei vorstellbar, $\mathrm{F}_{3}$ aufweist, so Cappelen, ist also optional: Die Überzeugungskraft von Chalmers Argument hängt nicht von einer solchen Annahme über den Status dieses Urteils ab. F3 hat in Chalmers' Argument somit kein wirksames Vorkommen. ${ }^{77}$

Dies ist nun wirklich eine abwegige Argumentation. Zunächst einmal können wir festhalten, dass das Urteil $\mathrm{P}_{7}$ für Chalmers' Argumentation von zentraler Wichtigkeit ist:

$\mathrm{P}_{7}$ : $\quad$ Eine Zombie-Welt (d. h. eine Welt, die physikalisch identisch mit unserer Welt ist, in der es aber kein Bewusstsein gibt) ist vorstellbar.

Gehen wir nachfolgend um des Argumentes willen einmal davon aus, $\mathrm{P}_{7}$ wäre begrifflich wahr. Wir hätten es dann mit dem folgenden Szenario zu tun: Ein Philosoph $A$ stützt sich auf ein Urteil ab, das die Eigenschaft $\mathrm{F}_{3}$ hat. Andere Philosophinnen und Philosophen lassen sich vom Argument von

76 Vgl. Cappelen 2012, S. 185, Chalmers 2002, S. 196.

77 Vgl. Cappelen 2012, S. 186f. 
$A$ überzeugen, sind aber irrtümlich der Ansicht, dass das relevante Urteil keine begriffliche Wahrheit ist, oder haben zum Status dieses Urteils keine Meinung. Sollten wir tatsächlich aufgrund der Existenz solcher verwirrten Philosophinnen und Philosophen zum Schluss zu kommen, es finde in einem entscheidenden Sinn keine Berufung auf eine begriffliche Wahrheit statt, da die Ansicht, das betreffende Urteil weise F3 auf, »optional« sei? Diese Haltung erscheint mir absurd.

An anderer Stelle führt Cappelen den für ihn relevanten Punkt wie folgt aus:

Keep in mind that the goal here is not to argue that there are no analytic or conceptual truths. The goal is to show that the thought experiments under consideration can be appreciated (and serve their argumentative purpose) even though we stay neutral on that issue. ${ }^{78}$

Nun ist aber in einem bestimmten Sinn das Funktionieren oder die Überzeugungskraft jedes Arguments unabhängig von der Frage, weshalb seine Prämissen wahr sind: Ein Argument ist grundsätzlich genau dann überzeugend für ein Subjekt $S$, wenn $S$ (a) die Prämissen für wahr und (b) das Argument für gültig hält. In Bezug darauf, was für die Wahrheit oder Falschheit der Prämissen eines Arguments ausschlaggebend ist, können wir also immer »neutral bleiben«, wenn wir seine Überzeugungskraft beurteilen. Und es ist immer möglich, dass jemand die Prämissen eines Arguments aus den falschen Gründen für wahr hält - ein Beispiel dafür sind Cappelen und Hawthorne, die im weiter oben besprochenen Fall das Urteil $\mathrm{P}_{5}$ offenbar unter anderem aus anthropologischen Gründen bejahen. Derartige Umstände erlauben aber natürlich keine Rückschlüsse darauf, aus welchen Gründen ein Urteil, auf das sich eine Philosophin oder ein Philosoph beruft, tatsächlich wahr beziehungsweise falsch ist. Die von Cappelen im obigen Zitat formulierte Erwägung ist also irrelevant dafür, ob in einem philosophischen Argument eine Abstützung auf ein F3-Urteil stattfindet, und (viii) ist für die Beantwortung dieser Frage kein geeigneter Anhaltspunkt.

\subsection{Fazit zur Abstützung auf $F_{3}$ in den Fallstudien}

Zur Rekapitulation: Ich habe im Abschnitt 4.1 neun Argumente aufgelistet, die Cappelen im Rahmen seiner Fallstudien dafür anführt, dass ein zur Diskussion stehendes Urteil nicht begrifflich wahr ist beziehungsweise dass in einem Argument keine Abstützung auf ein F3-Urteil stattfindet. Ich habe dafür argumentiert, dass die Argumente (vi) bis (viii) interessanter sind als (i) bis (v)

78 Cappelen 2012, S. 169, Fußnote 20. 
und (ix). (viii) hat sich jetzt auch als inadäquat herausgestellt, so dass nur (vi) und (vii) übrig bleiben:

(vi) Die Negation des relevanten Urteils bringt eine Möglichkeit zum Ausdruck.

(vii) Das relevante Urteil wird unter anderem auf der Grundlage empirischer Daten oder auf der Basis von Ergebnissen empirischer Wissenschaften gefällt.

Im Zusammenhang mit (vi) habe ich dafür argumentiert, dass es Cappelen in keinem der Fälle, in welchen er das Argument (vi) anführt, tatsächlich gelingt, zu zeigen, dass die Negation des betreffenden Urteils möglich ist. Im Zusammenhang mit (vii) hat sich herausgestellt, dass in zwei der drei Fällen, die Cappelen in diesem Kontext anführt, in Wirklichkeit keine Berufung auf empirische Befunde stattfindet. Im dritten Fall stützen sich die fraglichen Autoren tatsächlich nach eigenen Angaben auf empirische Daten ab, das relevante Urteil ist aber trotzdem, sofern es wahr ist, begrifflich wahr. Da es demnach möglich zu sein scheint, ein begrifflich wahres Urteil auf der Grundlage von empirischen Gründen zu fällen, stellt sich die Frage, ob (vii) überhaupt als Argument gegen das Vorliegen einer Abstützung auf eine begriffliche Wahrheit taugt. Diese Frage möchte ich hier allerdings offen lassen, da ihre Beantwortung mit einer Reihe von Komplikationen zusammenhängt, die weit über das Thema des vorliegenden Kapitels hinausgehen. ${ }^{79}$

\section{Gettier-Fälle}

Cappelen setzt es sich in Philosophy without Intuitions unter anderem zum Ziel, zu zeigen, dass es für die Methode möglicher Fälle nicht charakteristisch ist, dass eine Berufung auf Intuitionen oder auch nur auf begriffliche Wahrheiten stattfindet. Dabei prüft er hauptsächlich, wie bestimmte Philosophinnen und Philosophen ihr eigenes Unterfangen und die darin involvierten Urteile beurteilen, und macht sich nicht die Mühe, dafür zu argumentieren, dass die einzelnen besprochenen Urteile tatsächlich keines der von ihm identifizierten Intuitions-Merkmale aufweisen. Hierzu meint er berechtigt zu sein, da er sich ja nur für die empirische Frage interessiert, wie Philosophinnen und

79 Zum Beispiel: Wie hängen die Gründe dafür, dass ein Urteil wahr ist, mit den Gründen zusammen, die man dafür haben kann, das Urteil zu glauben? Und: Ließe sich dafür argumentieren, dass ich ein begrifflich wahres Urteil, das ich (ausschließlich) aus empirischen Gründen glaube und nicht auf der Grundlage einer Übersicht über die begrifflichen Zusammenhänge, nicht vollständig erfasst habe? 
Philosophen tatsächlich vorgehen, und nicht für irgendeine nicht-empirische Frage, mit der man sich im Lehnstuhl auseinandersetzen könnte oder müsste. Er moniert sogar in seiner Diskussion der Merkmale intuitiver Urteile, dass Philosophinnen und Philosophen mit anderen metaphilosophischen Auffassungen häufig Behauptungen aufstellen, die sich nicht empirisch überprüfen lassen:

[O]ften Centrality proponents will appeal to features that are practically impossible to detect and as a result their claims are empirically unsupportable. ${ }^{80}$

Dass sich die Behauptung, dass in der Philosophie häufig eine Abstützung beispielsweise - auf begriffliche Wahrheiten stattfindet, nicht ohne Weiteres empirisch überprüfen lässt, liegt allerdings bis zu einem bestimmten Grad in der Natur der Sache: Um festzustellen, ob eine Philosophin sich in einem bestimmten Argument auf eine begriffliche Wahrheit beruft, ist manchmal mehr Lehnstuhl-Arbeit als Beobachtung erforderlich. Denn wenn die Urteile, auf die eine Philosophin sich abstützt, erst einmal identifiziert sind (was vielleicht schon an und für sich nicht allein aufgrund von Beobachtung möglich ist), muss geprüft werden, ob eines dieser Urteile begrifflich wahr ist, und dies ist keine empirische Frage.

Ein Beispiel, das in Cappelens ausführlicher Diskussion der verschiedenen Gedankenexperimente fehlt, sind Gettier-Fälle. Cappelen begründet seine Entscheidung, diese Fälle nicht einzubeziehen, damit, dass ihnen in der Metaphilosophie und der Literatur um Intuitionen ohnehin bereits unangemessen viel Aufmerksamkeit geschenkt wird und dass in der aktuellen Debatte um Gettier-Fälle eine Fehlauffassung hinsichtlich dessen vorherrscht, wie der ursprüngliche Gettier-Fall funktioniert. Sollte sich herausstellen, so Cappelen, dass Gettier-Fälle ganz anders geartet sind als die Beispiele, die er in seinen Fallstudien bespricht, dann würde dies außerdem nur zeigen, dass GettierFälle nicht repräsentativ dafür sind, wie die Argumente der zeitgenössischen analytischen Philosophie funktionieren, und folglich auch nicht wichtig sind für die Fragestellung von Cappelens Buch. ${ }^{81}$

Da ich persönlich Gettier-Fälle für erhellende Beispiele für die Funktionsweise philosophischer Gedankenexperimente (auch von manchen, die Cappelen bespricht) halte, möchte ich kurz erwähnen, weshalb ich Cappelens

8o Cappelen 2012, S. 121.

81 Vgl. Cappelen 2012, S. 194. Der letzte Teil des Arguments gegen die Erwähnung von Gettier-Fällen wird bei Cappelen nur implizit zu verstehen gegeben. 
in einer Fußnote angedeutete Zurückweisung der Auffassung, es finde darin eine Berufung auf Intuitionen statt, als fehlgeleitet erachte. Cappelen schreibt:

Several points are worth noting about Gettier's original paper (1963). First, there is no talk of any intuitions about the case or about Gettier's inclinations to believe, his judgments or other psychological states. All he talks about is whether Smith knows. Second, when stating that Smith doesn't know in the first case, he immediately, in the next sentence, goes on to explain why. He says: "But is is equally clear that Smith does not know that (e) [The man who will get the job has ten coins in his pocket] is true; for (e) is true in virtue of the number of coins in Smith's pocket, while Smith does not know how many coins are in Smith's pocket, and bases his belief in (e) on a count of the coins in Jones's pocket, whom he falsely believes to be the man who will get the job" (p. 122). Gettier does not present the claim that Smith doesn't know as based on an intuition, he presents it as based on an argument. ${ }^{82}$

Dass Argumente für ein Urteil gegeben werden, führt Cappelen hier und an vielen anderen Stellen als Anhaltspunkt dafür an, dass das betreffende Urteil nicht die Eigenschaft F2 hat (die Eigenschaft, nicht begründungsbedürftig zu sein). Er formuliert diesen Punkt in einem anderen Kontext auch allgemein: Philosophische Fragen und im Speziellen auch Fragen über die richtige Beurteilung von Gedankenexperimenten bringen einen Rechtfertigungsanspruch (justification requirement) mit sich: Es genügt nicht, eine Frage wie »Ist personale Identität an psychologische Kontinuität geknüpft?« (Williams), »Gibt es ein Recht auf Abtreibung?«(Thomson), »Ist der Materialismus eine verteidigungsfähige Position? «(Chalmers), aber eben auch »Weiß Smith, dass die Person, die die Stelle bekommt, zehn Münzen in der Tasche hat? «(Gettier) mit »ja« oder »nein« zu beantworten, sondern eine entsprechende Antwort muss auch begründet werden. ${ }^{83}$ Vor diesem Hintergrund erscheint die These, dass philosophische Urteile F2 aufweisen, ganz allgemein als hoffnungslos.

$\mathrm{Zu}$ diesem Rechtfertigungsanspruch sind zwei Dinge zu sagen: Erstens: Die Begründung oder Rechtfertigung, die Gettier für sein Urteil anführt, dass Smith nicht weiß, dass die Person, die die Stelle bekommt, zehn Münzen in der Tasche hat, ist (ähnlich wie schon im weiter oben besprochenen Supermarkt-Fall von John Perry) Teil der Fallbeschreibung. Diese lautet im betreffenden GettierFall ungefähr wie folgt:

Smith und Jones haben sich beide für dieselbe Stelle beworben. Smith hat gute Gründe, anzunehmen, dass Jones die Stelle bekommt, (zum Beispiel weil der Chef der fraglichen Firma Smith gesagt hat, dass er Jones anstellen wird)

82 Cappelen 2012, S. 194, Fußnote 3. Eckige Klammern von Cappelen übernommen.

83 Vgl. Cappelen 2012, S. 135, 188 f. 
und er weiß, dass Jones zehn Münzen in der Tasche hat. Smith glaubt also, dass die Person, die die Stelle bekommen wird, zehn Münzen in der Tasche hat. Wie sich später herausstellen wird, bekommt Smith entgegen seiner Erwartung selbst die Stelle. Und auch er hat zehn Münzen in der Tasche, was ihm allerdings nicht bewusst ist. Also hat er die wahre, gerechtfertigte Überzeugung, dass die Person, die die Stelle bekommen wird, zehn Münzen in der Tasche hat. Aber weiß er, dass die Person, die die Stelle bekommen wird, zehn Münzen in der Tasche hat? ${ }^{84}$

Gettiers oben von Cappelen rekonstruierte Antwort auf diese Frage lautet: Nein, Smith weiß es nicht, denn dass die Person, die die Stelle bekommen wird, zehn Münzen in der Tasche hat, ist ja wahr, weil Smith die Stelle bekommen wird und zehn Münzen in der Tasche hat, was Smith aber selbst gar nicht weiß. Er glaubt ja, dass Jones die Stelle bekommen wird, von dem er weiß, dass er zehn Münzen in der Tasche hat. Damit wiederholt Gettier aber nur die für seine Beantwortung der Frage relevanten Aspekte des Falls. Er führt keine neuen, von der Fallbeschreibung nicht begrifflich implizierten Aspekte an, wie es beispielsweise im Fall von Sick oder Libro (S. 226f. des vorliegenden Kapitels) passieren müsste. Dass Gettier die relevanten Aspekte des Falls zur Begründung seines Urteils wiederholt, ist kein Argument dagegen, dass er sein Urteil ausschließlich auf der Grundlage seiner Begriffskompetenz fällt und dass es deshalb in einem bestimmten Sinn nicht begründungsbedürftig ist. Gettier könnte auch sagen: »Da der Fall so-und-so charakterisiert ist, fällt er nicht unter den-und-den Begriff«. Und als »Begründung « könnte er sagen: So verwenden wir eben den Ausdruck »wissen «. Nichts von dem, was Gettier schreibt, steht mit der Hypothese im Widerspruch, dass das von ihm gefällte Urteil über Smith F2 aufweist.

Zweitens: Wenn wir von der Beurteilung einzelner Fallbeispiele absehen und Fragen betrachten wie »Ist personale Identität an psychologische Kontinuität geknüpft?«, »Gibt es ein Recht auf Abtreibung?« oder »Ist der Materialismus eine verteidigungsfähige Position? «, dann verhält es sich anders als im vorangehend besprochenen Fall. Antworten auf diese Frage sind in der Tat rechtfertigungsbedürftig, und zu ihrer Rechtfertigung können nicht einfach Aspekte der Fragestellung wiederholt werden. In die Reihe solcher Fragen würde auch gehören: »Ist Wissen dasselbe wie wahre, gerechtfertigte Überzeugung?«. Und zur Beantwortung dieser Frage hat Gettier den Fall um die Münzen in der Tasche von Smith angeführt. Das Urteil, dass Smith im oben beschriebenen Fall über eine wahre gerechtfertigte Überzeugung, aber nicht über Wissen verfügt, ist nicht begründungsbedürftig und dient als Begründung für die Verneinung

$84 \quad$ Vgl. Gettier 1963, S. 122. 
der Frage, ob Wissen dasselbe wie wahre, gerechtfertigte Überzeugung ist. Die »großen « Fragen, für die sich Philosophinnen und Philosophen eigentlich interessieren, gehen also in der Tat mit einem Rechtfertigungsanspruch einher. Aber dasselbe gilt nicht zwingend für Antworten auf die Fragen betreffend die Gedankenexperimente, die zur Rechtfertigung einer Antwort auf eine dieser großen Frage, angeführt werden.

Die Irrelevanz der Begriffsanalyse

Cappelen zufolge sind Vertreterinnen und Vertreter von Centrality häufig auch der Auffassung, dass die Begriffsanalyse in der Philosophie eine wichtige Rolle spielt, und umgekehrt:

Those who think philosophers appeal to intuitions when they make judgments about cases also tend to see philosophers as engaged in conceptual analysis when they appeal to cases and vice versa. ${ }^{85}$

Meines Erachtens stimmt zumindest das »vice versa «ier nicht. Unter den Philosophinnen und Philosophen, die im Anschluss an den späten Wittgenstein eine begriffsanalytische Philosophie-Konzeption verteidigt haben, gibt es beispielsweise höchstens einige wenige, die von sich selbst sagen würden, dass sie sich in irgendeinem Sinn des Wortes auf »Intuitionen « berufen. Und mit Blick auf Cappelens drei Intuitions-Merkmale lässt sich Folgendes sagen: Für die begriffsanalytische Philosophie, wie sie im vorliegenden Buch verteidigt wird, sind natürlich Urteile mit dem Merkmal F3 relevant. Zumindest triviale begriffliche Wahrheiten verfügen außerdem über das Merkmal F2: Sie lassen sich nicht begründen, oder höchstens mit dem Hinweis: »Das ist eben die Art, wie wir die betreffenden Wörter verwenden«, und dies entspricht weniger einer Begründung als einer Zurückweisung der Aufforderung zur Begründung. F1 schließlich, das Merkmal der besonderen Phänomenologie bestimmter Urteile, spielt bei der vom späten Wittgenstein inspirierten begriffsanalytischen Philosophie überhaupt keine Rolle.

Mit »Conceptual Analysis and Intuitions« widmet Cappelen ein ganzes Kapitel seiner Monographie dem Thema Begriffsanalyse. In diesem Kapitel argumentiert er dafür, dass die Auffassung, dass Philosophinnen und Philosophen Begriffsanalyse betreiben sollten und dass sie sich dabei auf Intuitionen

85 Cappelen 2012, S. 205f. 
berufen müssen, ${ }^{86}$ drei Herausforderungen ausgesetzt ist, die nur schwer oder überhaupt nicht zu meistern sind:

1. Die Herausforderung, zu zeigen, dass der Begriff der Begriffsanalyse und damit verbundene Begriffe wie begriffliche Möglichkeit und begriffliche Notwendigkeit überhaupt kohärent sind.

2. Angenommen, dass es begriffliche Wahrheiten gibt:Die Herausforderung, zu zeigen, dass solche Wahrheiten für die Philosophie relevant sind.

3. Angenommen, dass der Begriff Begriffsanalyse kohärent ist: Die Herausforderung, zu zeigen, dass Intuitionen für die Begriffsanalyse einen evidenziellen Wert haben. ${ }^{87}$

Im Zusammenhang mit (1) schreibt Cappelen, Williamson 2007 habe überzeugend gegen die Existenz begrifflicher Wahrheiten argumentiert und wer die Position verteidigen wolle, dass Begriffsanalysen möglich sind, schulde uns eine stichhaltige Erwiderung auf Williamson. ${ }^{88}$ Einige stichhaltige Argumente gegen Williamson wurden beispielsweise in Glock 2010, Hacker 2009a und Schroeder 2009 bereits geliefert. Und ich selbst bin auf die Schwächen von Williamsons Argumentation in Bezug auf begriffliche Wahrheiten ausführlich im Kapitel 5 des vorliegenden Buchs eingegangen.

Die Herausforderung (2), zu zeigen, dass begriffliche Wahrheiten für die Philosophie relevant sind, unterteilt Cappelen in drei Teil-Herausforderungen:

2a. Die Herausforderung der Ausweitung: Wenn wir um des Arguments willen einräumen, dass beispielsweise der Satz »Wenn es wahr ist, dass $p$, und wahr ist, dass wenn $p$, dann $q$, dann ist es auch wahr, dass $q$ « begrifflich wahr ist, dann sind wir immer noch weit davon entfernt, gezeigt zu haben, dass es interessante begriffliche Wahrheiten gibt, die beispielsweise relevant sind für die Beantwortung der philosophischen Fragen, was Wahrheit, Freiheit, Wissen, Bedeutung, Kausalität, Personen oder Bewusstsein sind. ${ }^{89}$

86 Eigentlich charakterisiert Cappelen die relevante Auffassung wie folgt: »that if someone were to engage in conceptual analyis, then she would have to rely on intuitions«. Es ist aber nicht klar, weshalb sich für jemanden, der diese Position vertritt, die Herausforderung (2) ergeben sollte. Ich habe deshalb die Charakterisierung der zur Diskussion stehenden Position um eine Komponente erweitert.

87 Vgl. Cappelen 2012, S. 206.

88 Vgl. Cappelen 2012, S. 210f.

89 Vgl. Cappelen 2012, S. 212f. Cappelen drückt sich in diesem Kontext ein bisschen problematisch aus, indem er verlangt, dass gezeigt werde, dass es unter den begrifflichen Wahrheiten unter anderem auch »interesting and substantive philosophical claims « gebe (vgl. Cappelen 2012, S. 213; Cappelens Hervorhebung durch meine eigene ersetzt). Es ist unklar, was Cappelen hier mit »substantive« meint, aber so, wie ich das Wort verstehe, ist es bei substanziellen Urteilen per definitionem ausgeschlossen, dass sie begrifflich wahr sind (vgl. dazu auch die Abschnitte 2.6 und 3.2 des Kapitels 5 des vorliegenden Buchs). 
2b. Die Herausforderung des Erfolgsnachweises: Welche Methoden sich dafür eignen, philosophische Probleme zu lösen, ist, so Cappelen, eine empirische Frage. Von jemandem, der behauptet, die Begriffsanalyse sei eine geeignete Methode, können wir also erwarten, dass er empirische Belege dafür anführt, dass diese Methode in der Vergangenheit zu philosophischem Fortschritt geführt hat. ${ }^{90}$

2c. Die Herausforderung der Motivation: Die Verteidigerin oder der Verteidiger der Begriffsanalyse schuldet uns Cappelen zufolge im Übrigen eine Erklärung dafür, dass wir uns überhaupt für begriffliche Wahrheiten interessieren sollten. ${ }^{91}$

Diese drei Herausforderungen lassen sich nicht sinnvoll voneinander trennen: Wenn in Reaktion auf (2a) gezeigt werden kann, dass begriffliche Fragen für die Beantwortung philosophischer Fragen relevant sind, dann hat sich die Herausforderung (2c) damit ebenfalls erledigt: Wir sollten uns für begriffliche Wahrheiten interessieren, weil sie für die Beantwortung philosophischer Fragen relevant sind. Und wenn in Reaktion auf (2b) gezeigt werden kann, dass die Begriffsanalyse in der Vergangenheit zu philosophischem Fortschritt geführt hat, beantwortet sich die Frage (2c), weshalb wir uns für begriffliche Wahrheiten interessieren sollten, ebenfalls von selbst. Der Grund dafür, dass Cappelen (2c) separat anführt, hängt wahrscheinlich damit zusammen, dass er davon ausgeht, dass (2a) und ( $2 \mathrm{~b}$ ) nicht gemeistert werden können: Wenn es keine philosophisch relevanten begrifflichen Wahrheiten und auch keine Belege für den Erfolg der Begriffsanalyse gibt, wieso sollten wir uns dann für begriffliche Wahrheiten interessieren?

Cappelen macht vier Vorschläge dazu, was Verteidigerinnen und Verteidiger der Begriffsanalyse als Motivation dafür angeben könnten, sich mit begrifflichen Wahrheiten auseinanderzusetzen: Sie könnten sagen, dass begriffliche Wahrheiten für sie deshalb interessant sind, (i) weil sie notwendig sind, (ii) weil sie a priori sind oder (iii) weil sie das Fundament darstellen, auf dem unser restliches (philosophisches) Wissen aufgebaut ist. Schließlich könnten sie auch (iv) geltend machen, dass ihr Interesse für begriffliche Wahrheiten ebenso wenig einer Rechtfertigung bedarf wie etwa das Interesse mancher Leute an mittelalterlicher Kartografie..$^{92}$

Keiner dieser Gründe entspricht der Motivation für mein Interesse an begrifflichen Wahrheiten. Meine Antwort auf (2c) lautet: Begriffliche Wahrheiten sind für die Philosophie deshalb interessant, weil viele philosophische

9o Vgl. Cappelen 2012, S. 214.

91 Vgl. Cappelen 2012, S. 214f.

92 Vgl. Cappelen 2012, S. 215f. 
Probleme entweder begriffliche Probleme sind (wie etwa »Was ist Wissen?«) oder auf begrifflicher Verwirrung beruhen (wie etwa »Wie kann Achilles die Schildkröte überholen?«). Diese Probleme können wir nur lösen oder überwinden, indem wir Klarheit über die jeweils relevanten begrifflichen $\mathrm{Zu}$ sammenhänge erlangen. Wenn gezeigt werden kann, dass philosophische Probleme tatsächlich oft auf diese Weise mit begrifflichen Zusammenhängen verknüpft sind, dann erübrigt sich die Herausforderung (2b): Dass ein begriffliches Problem am besten durch eine Untersuchung der entsprechenden Begriffe gelöst werden kann, ist keine empirische Behauptung und muss folglich auch nicht empirisch belegt werden. Natürlich können aber Beispiele begriffsanalytischer Philosophie gegeben werden, die zumindest in Teilen der Philosophie zu Fortschritt geführt haben, indem sie dabei geholfen haben, Verwirrungen zu überwinden und begriffliche Zusammenhänge deutlich zu machen. Etwa Wittgensteins Philosophische Untersuchungen, Gilbert Ryles The Concept of Mind, Strawsons Individuals, Austins Sense and Sensibilia, P. M. S. Hackers Appearance and Reality und Bede Rundles Time, Space, and Metaphysics.

(2a) ist eine interessante Herausforderung. Cappelen hat Recht damit, dass es ein großer Schritt ist von Sätzen wie »Wenn es wahr ist, dass $p$, und wahr ist, dass wenn $p$, dann $q$, dann ist es auch wahr, dass $q$ « zu philosophischen Erkenntnissen über Wahrheit, Freiheit, Wissen, Bedeutung, Kausalität, Personen oder Bewusstsein. Und interessante philosophische Erkenntnisse werden natürlich nicht von Sätzen wie »Rot ist eine Farbe« oder »Junggesellen sind unverheiratet« ausgedrückt. Der springende Punkt, für den ich im vorliegenden Buch bereits an verschiedener Stelle argumentiert habe, ist, dass es auch synoptische begriffliche Wahrheiten wie »Es kann keine private Sprache geben « oder »Es stimmt nicht, dass ein Urteil genau dann wahr ist, wenn es Teil eines kohärenten Systems von Urteilen ist « gibt, die auf triviale begriffliche Wahrheiten zurückgeführt werden können.

Bleibt noch die Herausforderung (3): Wieso sollten wir annehmen, dass wir bei der Begriffsanalyse auf Intuitionen zurückgreifen müssen? Wenn Intuitionen Urteile mit einer besonderen Phänomenologie sind, vertrete ich nicht die Auffassung, dass wir dies müssen. Wir müssen uns allerdings auf Urteile mit den Intuitions-Merkmalen F2 und F3 abstützen, die ich (im Gegensatz zu bestimmten anderen Vertreterinnen und Vertretern einer begriffsanalytischen Philosophie ${ }^{93}$ ) nicht als »Intuitionen « bezeichnen würde.

Cappelen macht geltend, die Antwort auf die Frage, ob wir bei der Begriffsanalyse auf Intuitionen zurückgreifen müssen, hänge zunächst einmal davon 
ab, was Begriffe seien. Und diese Frage werde von allen ernstzunehmenden Teilnehmerinnen und Teilnehmern der entsprechenden Debatte als mindestens partiell empirische Frage behandelt. Auch die Frage, ob Intuitionen bei der Analyse von Begriffen eine wichtige Rolle spielen, sei eine empirische Frage. Und wenn wir davon ausgehen, dass Begriffe »psychologisch real« seien und es sich bei ihnen beispielsweise um Repräsentationen im Gehirn von Sprecherinnen und Sprechern handle, sei zumindest auf den ersten Blick nicht klar, weshalb wir sie durch eine Lehnstuhl-Aktivität wie das Abfragen von Intuitionen analysieren können sollten. ${ }^{94} \mathrm{Ganz}$ in diesem Sinne schreibt Cappelen auch an anderer Stelle:

[I]f someone really is interested in conceptual truths [...] then she should be engaged deeply in collaborative work with empirical scientists working on concept possession, concept acquisition and a range of related topics. ${ }^{95}$

Mit keinem dieser Punkte bin ich einverstanden. Erstens hängt die Frage, ob wir uns bei der Begriffsanalyse auf Intuitionen berufen müssen, weniger von der Frage ab, was Begriffe sind, als von der Frage, was Begriffsanalyse ist. Cappelen meint in diesem Kontext auch: »no one can seriously engage in an activity labeled $>$ conceptual analysis $<$ without a theory of what concepts are «, 96 und dem möchte ich ebenfalls widersprechen. Eine Reihe von Philosophinnen und Philosophen können sich völlig einig darüber sein, dass »männliche Ente« eine adäquate Analyse des Begriffs Enterich ist, ohne sich im Geringsten darüber zu verständigen, was Begriffe sind, oder auch nur eine Meinung zu dieser Frage zu haben. Und auch beispielsweise in der Debatte darum, wie wir die Analyse von Wissen als wahre, gerechtfertigte Überzeugung ergänzen oder modifizieren können, damit sie unserem gewöhnlichen Wissensbegriff gerecht wird, konnten Vertreterinnen und Vertreter ganz unterschiedlicher Auffassungen darüber, was Begriffe sind, miteinander diskutieren, ohne aneinander vorbeizureden. Um zu verstehen, was es bedeutet, Begriffe zu analysieren, und um dazu in der Lage sein, Analysen vorzuschlagen, zu testen und zu kritisieren, benötigt man keine Theorie darüber, was Begriffe sind.

Zweitens ist die Frage, was Begriffe sind, keine empirische Frage, sondern die Frage nach einer Klärung des Begriffs Begriff. Und es gibt pace Cappelen auch ernstzunehmende Teilnehmerinnen und Teilnehmer an der entsprechenden

94 Vgl. Cappelen 2012, S. 207-209.

95 Cappelen 2012, S. 216.

96 Cappelen 2012, S. 207. 
Debatte, welche die Frage, was Begriffe sind, nicht als (partiell) empirische Frage behandeln. ${ }^{97}$

Drittens: Ist die Frage, ob Intuitionen bei der Analyse von Begriffen eine wichtige Rolle spielen, eine empirische Frage? Das kommt darauf an, wie wir sie verstehen. Wir können sie als Frage auffassen, die auf die gängige Praxis der Analyse von Begriffen gerichtet ist: Wie gehen Philosophinnen und Philosophen tatsächlich vor, wenn sie Begriffe analysieren? Berufen sie sich auf Intuitionen? Dann ist es eine empirische Frage. Oder wir können sie normativ auffassen: Wie sollte man bei der Analyse von Begriffen vorgehen? Ist es zweckmäßig, Intuitionen heranzuziehen? Dann ist es keine empirische Frage. Für das Thema, das Cappelen im betreffenden Kapitel seines Buchs bespricht, nämlich welche Antworten uns Philosophinnen und Philosophen schuldig sind, die behaupten, dass die Begriffsanalyse eine für die Philosophie wichtige Methode ist und dass das Betreiben von Begriffsanalyse eine Abstützung auf Intuitionen erfordert, ist die zweite, nicht empirische Frage interessanter als die erste.

Viertens und letztens: Die Ansicht, dass Begriffe »psychologisch real« im von Cappelen angedeuteten Sinn sind, Repräsentationen im Gehirn von Sprecherinnen und Sprechern, die in kausale Beziehungen eingehen können, halte ich für falsch. ${ }^{98}$ Ungeachtet dessen, verstehe ich nicht, wie die $\mathrm{Zu}$ sammenarbeit mit empirischen Wissenschaftlerinnen und Wissenschaftlern, die Forschung in den Bereichen Begriffsbesitz, Begriffsaneignung usw. betreiben, für Philosophinnen und Philosophen fruchtbar sein sollte, die Begriffe für Repräsentationen im Gehirn halten und sich zum Beispiel für den Begriff der Wahrheit, der Gerechtigkeit oder der Person interessieren. Es ist nicht ersichtlich, wie eine Wissenschaftlerin, die sich damit auskennt, welche neuronalen Zustände mit dem Besitz von Begriffen assoziiert sind oder was im Gehirn passiert, wenn wir uns einen neuen Begriff aneignen, uns dabei helfen sollte, die Frage zu beantworten, was »Wahrheit« bedeutet oder wie der Begriff der Gerechtigkeit zu analysieren ist.

Es ist also keine der drei Herausforderungen, mit denen Vertreterinnen und Vertreter der Auffassung, dass wir in der Philosophie Begriffe analysieren sollten, Cappelen zufolge konfrontiert sind, unüberwindbar.

97 Z. B. Hans-Johann Glock oder P.M.S. Hacker, aber auch Michael Dummett und Christopher Peacocke.

98 Vgl. für entsprechende Argumente z. B. Hacker 1987, S. 497-499, Glock 2006, S. 46-48 und Kenny 2010. 
Ich habe im vorliegenden Kapitel dafür argumentiert, dass es Cappelen in Philosophy without Intuitions nicht gelingt, zu zeigen, dass begriffliche Wahrheiten für die Philosophie von keiner besonderen Relevanz sind. Dabei habe ich einerseits Cappelens Kriterium des wirksamen Vorkommens von Intuitions-Merkmalen kritisiert, andererseits aber auch versucht, zu zeigen, dass in manchen von Cappelens Fallstudien begriffliche Wahrheiten in der Tat eine wichtige Rolle spielen und dass einige Anhaltspunkte, die Cappelen dafür verwendet, eine Berufung auf begriffliche Wahrheiten auszuschließen, zu diesem Zweck ungeeignet sind. Des Weiteren habe ich versucht, zu zeigen, dass Cappelen in seinen Ausführungen zum Anspruch auf Rechtfertigung, der ihm zufolge mit jeder philosophischen Behauptung einhergeht, zu wenig zwischen allgemeinen Aussagen darüber, was beispielsweise Wissen ist, und spezifischen Urteilen in Bezug auf Gedankenexperimente unterscheidet. Zum Schluss des Kapitels habe ich dafür argumentiert, dass jede der drei Herausforderungen gemeistert werden kann, mit denen Cappelen zufolge Philosophinnen und Philosophen konfrontiert sind, wenn sie glauben, dass man in der Philosophie Begriffsanalyse betreiben sollte.

Wie bereits erwähnt, finden sich in Philosophy without Intuitions Behauptungen wie »In the case studies that I investigate [...], we will consistently find an absence of all of $\mathrm{F}_{1}-\mathrm{F}_{3}{ }{ }^{99}$ oder »[E]ven if there are conceptual a priori truths, exemplified by >All vixens are female foxes $<$, those are irrelevant to philosophical practice $«{ }^{100}$ Diese Behauptungen habe ich oben bereits entkräftet. Die These, die Cappelen zu Beginn seines Buchs als zentralen Gegenstand seiner Kritik identifiziert, besteht allerdings nicht darin, dass begriffliche Wahrheiten (oder Urteile mit den anderen beiden Intuitions-Merkmalen) in philosophischen Argumenten vorkommen oder dass sie relevant für die philosophische Praxis sind, sondern lautet: »Contemporary analytic philosophers rely on intuitions as evidence (or as a source of evidence) for philosophical theories. «101 Und während es sicherlich stimmt, dass begriffliche Wahrheiten in der Philosophie eine wichtige Rolle spielen, ist es zweifelhaft, ob es adäquat ist, zu sagen, dass sie die Rolle von Evidenz spielen.

Diese Frage hängt natürlich davon ab, mit welchem Begriff der Evidenz wir operieren. Was Evidenz ist, ist unter Epistemologinnen und Epistemologen umstritten, und verschiedene Konzeptionen von Evidenz passen unterschiedlich

99 Cappelen 2012, S. 114.

100 Cappelen 2012, S. 202.

101 Cappelen 2012, S. 3. Hervorhebung von mir. 
gut zur Auffassung begrifflicher Wahrheiten als Evidenz in der Philosophie. Im Oxford Dictionary of Philosophy definiert Simon Blackburn Evidenz als »[t]hat which raises or lowers the probability of a proposition«. In diesem Sinn fungieren begriffliche Wahrheiten natürlich nicht als Evidenz für philosophische Wahrheiten. In dem Maße, in dem philosophische Wahrheiten begrifflich wahr sind, haben sie nämlich die Wahrscheinlichkeit 1 (bzw. 100\%), und andere begriffliche Wahrheiten können diese Wahrscheinlichkeit nicht erhöhen. Wenn wir »Evidenz für die Proposition $p$ « andererseits weiter fassen und im Sinne von »Grund dafür, $p$ zu glauben« verstehen, dann können wir eventuell einräumen, dass begriffliche Wahrheiten als Evidenz für andere begriffliche Wahrheiten fungieren können. Wenn wir so reden wollen, müssen wir aber wohl auch akzeptieren, dass ein mathematischer Beweis Evidenz für die entsprechende mathematische Wahrheit ist, was sich zumindest mit meinem Sprachgefühl nicht ohne Weiteres vereinbaren lässt. Relevant für Cappelens Argumentation ist meine Kritik in den vorangehenden Abschnitten aber unabhängig davon, welche Entscheidungen wir in Bezug auf den Gebrauch des Wortes »Evidenz« treffen. 